University of Wollongong

Research Online

Faculty of Engineering and Information

Faculty of Engineering and Information

Sciences - Papers: Part A

Sciences

$1-1-2016$

Nonlinear dynamical behavior of axially accelerating beams: threedimensional analysis

Mergen H. Ghayesh

University of Wollongong, mergen@uow.edu.au

Hamed Farokhi

McGill University

Follow this and additional works at: https://ro.uow.edu.au/eispapers

Part of the Engineering Commons, and the Science and Technology Studies Commons

Research Online is the open access institutional repository for the University of Wollongong. For further information contact the UOW Library: research-pubs@uow.edu.au 


\title{
Nonlinear dynamical behavior of axially accelerating beams: three-dimensional analysis
}

\author{
Abstract \\ The three-dimensional (3D) nonlinear dynamics of an axially accelerating beam is examined numerically \\ taking into account all of the longitudinal, transverse, and lateral displacements and inertia. Hamilton's \\ principle is employed in order to derive the nonlinear partial differential equations governing the \\ longitudinal, transverse, and lateral motions. These equations are transformed into a set of nonlinear \\ ordinary differential equations by means of the Galerkin discretization technique. The nonlinear global \\ dynamics of the system is then examined by time-integrating the discretized equations of motion. The \\ results are presented in the form of bifurcation diagrams of Poincare maps, time histories, phase-plane \\ portraits, Poincare sections, and fast Fourier transforms (FFTs).
}

\section{Keywords}

nonlinear, dimensional, dynamical, analysis, behavior, axially, accelerating, beams, three

Disciplines

Engineering | Science and Technology Studies

\section{Publication Details}

Ghayesh, M. H. \& Farokhi, H. (2016). Nonlinear dynamical behavior of axially accelerating beams: threedimensional analysis. Journal of Computational and Nonlinear Dynamics, 11 (1), 011010-1-011010-16. 


\author{
Mergen H. Ghayesh ${ }^{1}$ \\ School of Mechanical, \\ Materials and Mechatronic Engineering, \\ University of Wollongong, \\ New South Wales 2522, Australia \\ e-mail: mergen@uow.edu.au \\ Hamed Farokhi \\ Department of Mechanical Engineering, \\ McGill University, \\ Montreal, QC H3A OC3, Canada \\ e-mail: hamed.farokhi@mail.mcgill.ca
}

\section{Nonlinear Dynamical Behavior of Axially Accelerating Beams: Three-Dimensional Analysis}

\begin{abstract}
The three-dimensional (3D) nonlinear dynamics of an axially accelerating beam is examined numerically taking into account all of the longitudinal, transverse, and lateral displacements and inertia. Hamilton's principle is employed in order to derive the nonlinear partial differential equations governing the longitudinal, transverse, and lateral motions. These equations are transformed into a set of nonlinear ordinary differential equations by means of the Galerkin discretization technique. The nonlinear global dynamics of the system is then examined by time-integrating the discretized equations of motion. The results are presented in the form of bifurcation diagrams of Poincaré maps, time histories, phase-plane portraits, Poincaré sections, and fast Fourier transforms (FFTs).
\end{abstract} [DOI: 10.1115/1.4029905]

Keywords: axially accelerating beams, bifurcation diagrams, three-dimensional (3D) modeling, nonlinear dynamical behavior

\section{Introduction}

Many systems in mechanical and industrial applications, such as band saw blades, power transmission chains and belts, robotic manipulators, conveyor belts, and cable tramways, can be modeled as axially moving continua [1-6]. One of the interesting features of such systems is the presence of the axial speed, which renders the system gyroscopic; as a result, traveling waves arise along the beam and the flexural stiffness of the beam is weakened. The widespread applications of these systems along with their complex and interesting dynamics motivated an extensive amount of research on this topic over the last few decades.

In general, in real-life applications, the presence of unwanted imperfections due to either geometrical or dynamical sources results in an axial acceleration. Hence, it is more practical to model the axial speed as a time-dependent quantity [7,8]; here, the axial speed comprises a constant mean value along with a harmonic fluctuating component, which generates an axial acceleration in the system.

The literature concerning the dynamics of axially moving beams with constant axial speed is quite large [9-11]. For example, Sze et al. [12] and Huang et al. [13] investigated the subcritical resonant dynamic response of an axially moving beam by means of the incremental harmonic balance method. Wickert [14] examined the nonlinear dynamics of an axially moving tensioned beam in both sub- and supercritical axial speed regimes. Marynowski and Kapitaniak [15] introduced different energy dissipation mechanisms, such as Kelvin-Voigt and Zener, in the mathematical model of axially moving beams, and solved the equations numerically. Chen and Yang [16] examined the dynamics and stability of an axially moving viscoelastic beam with hybrid supports, employing the Kelvin-Voigt viscoelastic model and the method of multiple scales. Riedel and Tan [17] examined the coupled forced dynamic response of an axially moving beam in the presence of a three-to-one internal resonance between the first two transverse modes. Ghayesh [18] analyzed the dynamic

${ }^{1}$ Corresponding author

Contributed by the Design Engineering Division of ASME for publication in the Journal of Computational and Nonlinear Dynamics. Manuscript received December 15, 2014; final manuscript received February 14, 2015; published online June 30, 2015. Assoc. Editor: Stefano Lenci. stability and bifurcations of an axially moving beam with an intermediate spring-support.

The literature concerning the linear and nonlinear planar (i.e., taking into account only the in-plane displacements) dynamics of axially moving beams with time-variant axial speed is quite extensive. For instance, Suweken and Van Horssen [19] contributed to the field by analyzing the transverse dynamics of a beamlike conveyor belt with a time-dependent axial speed, by means of the method of multiple scales. Pakdemirli and coworkers [20-22] employed different analytical techniques, such the method of multiple scales and matched asymptotic expansions, in order to examine the dynamics of axially accelerated beams. The incremental harmonic balance technique was employed by $\mathrm{Xu}$ and $\mathrm{Zhu}$ [23], who investigated the periodic response of high-dimensional models of an axially moving beam. Ghayesh $[24,25]$ contributed to the field by investigating the transverse and coupled longitudinal-transverse nonlinear dynamics of axially accelerating beams. All of the aforementioned valuable studies neglect the effect of the lateral motion on the nonlinear dynamics of axially accelerating beams.

Contributions of the current study to the field: The present study, for the first time, analyzes the coupled global, 3D nonlinear dynamical behavior of an axially accelerating beam by retaining the longitudinal, transverse, and lateral displacements and inertia. The nonlinear partial differential equations are transformed into a high-dimensional discretized model by means of the Galerkin technique; it should be noted that solving such a high-dimensional system requires a significant amount of computations and thus the computer codes should be welloptimized for both run-time and accuracy. The discretized equations of motion are solved numerically via direct time integration, resulting in time traces and hence the bifurcation diagrams of Poincaré maps. The results are also presented in the form of time histories, phase-plane portraits, Poincaré sections, and FFTs.

\section{Model Development}

Shown in Fig. 1 is the schematic representation of the system, i.e., a hinged-hinged beam of length $L$, axial stiffness $E A$, and flexural rigidity $E I$. The beam is under a pretention $p$ in the $x$-direction and traveling at a time-dependent axial speed $c(t)$. The 


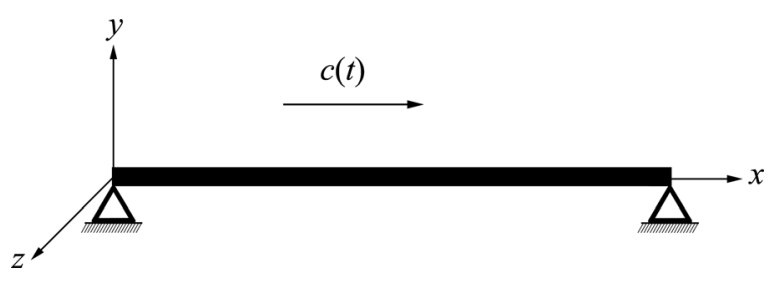

Fig. 1 Schematic representation of a 3D axially accelerating beam

longitudinal, transverse, and lateral displacements, in the $x, y$, and $z$ directions, respectively, are represented by $u(x, t), w(x, t)$, and $v(x, t)$, respectively; $x$ denotes the axial coordinate.

The nonlinear partial differential equations of motion are derived assuming that: (1) shear deformation and rotary inertia are neglected, i.e., the Euler-Bernoulli beam theory is employed [26-29]; (2) the nonlinear behavior is due to the stretching effect of the midplane of the beam; (3) the beam is under a constant pretension $p$ in the axial direction; (4) the cross section of the beam is uniform along the entire length [30,31]; and (5) the rotation of beam cross section is assumed to be small, which neglects the nonlinearities in the curvature-displacement relation [32-39].

The kinetic energy of the system can be formulated as

$$
\begin{aligned}
T= & \frac{1}{2} \rho A \int_{0}^{L}\left\{\left[\frac{\partial u}{\partial t}+c(t)\left(1+\frac{\partial u}{\partial x}\right)\right]^{2}+\left(\frac{\partial v}{\partial t}+c(t) \frac{\partial v}{\partial x}\right)^{2}\right. \\
& \left.+\left(\frac{\partial w}{\partial t}+c(t) \frac{\partial w}{\partial x}\right)^{2}\right\} \mathrm{d} x
\end{aligned}
$$

where $c(t)$ represents the time-dependent axial speed which is in the form of $c_{0}+c_{1} \sin (\omega t) ; \omega$ denotes the frequency of speed variations.

The nonlinear strain-displacement relation for a $3 \mathrm{D}$ Euler-Bernoulli beam model can be expressed as

$$
\varepsilon_{x}=\frac{\partial u}{\partial x}+\frac{1}{2}\left(\frac{\partial v}{\partial x}\right)^{2}+\frac{1}{2}\left(\frac{\partial w}{\partial x}\right)^{2}-z \frac{\partial^{2} v}{\partial x^{2}}-y \frac{\partial^{2} w}{\partial x^{2}}
$$

where $\varepsilon_{x}$ denotes the axial strain of an arbitrary point of the beam located at distances $y$ and $z$ in the $y$ and $z$ directions from the centerline of the beam.

The potential energy of the system is given by

$$
\begin{aligned}
U= & \int_{0}^{L}\left\{p\left[\frac{\partial u}{\partial x}+\frac{1}{2}\left(\frac{\partial v}{\partial x}\right)^{2}+\frac{1}{2}\left(\frac{\partial w}{\partial x}\right)^{2}\right]+\frac{1}{2} E A\left[\frac{\partial u}{\partial x}+\frac{1}{2}\left(\frac{\partial v}{\partial x}\right)^{2}\right.\right. \\
& \left.\left.+\frac{1}{2}\left(\frac{\partial w}{\partial x}\right)^{2}\right]^{2}\right\} \mathrm{d} x+\frac{1}{2} \int_{0}^{L} E\left[I_{y}\left(\frac{\partial^{2} v}{\partial x^{2}}\right)^{2}+I_{z}\left(\frac{\partial^{2} w}{\partial x^{2}}\right)^{2}\right] \mathrm{d} x
\end{aligned}
$$

where $I_{y}$ and $I_{z}$ stand for the area moment of inertia about the $y$ and $z$ axes, respectively.

The variation of the work of damping due to a medium moving at the same speed of the beam can be written as

$$
\delta W_{D}=-\mu \int_{0}^{L}\left(\frac{\partial u}{\partial t} \delta u+\frac{\partial v}{\partial t} \delta v+\frac{\partial w}{\partial t} \delta w\right) \mathrm{d} x
$$

Inserting Eqs. (1)-(4) into the extended Hamilton's principle yields the following three nonlinear partial differential equations of motion:

$$
\begin{gathered}
\rho A\left(\frac{\partial^{2} u}{\partial t^{2}}+2 c(t) \frac{\partial^{2} u}{\partial x \partial t}+\frac{\mathrm{d} c(t)}{\mathrm{d} t}\left(1+\frac{\partial u}{\partial x}\right)+c(t)^{2} \frac{\partial^{2} u}{\partial x^{2}}\right)-E A\left(\frac{\partial^{2} u}{\partial x^{2}}+\frac{\partial v}{\partial x} \frac{\partial^{2} v}{\partial x^{2}}+\frac{\partial w}{\partial x} \frac{\partial^{2} w}{\partial x^{2}}\right)+\mu \frac{\partial u}{\partial t}=0 \\
\rho A\left(\frac{\partial^{2} w}{\partial t^{2}}+2 c(t) \frac{\partial^{2} w}{\partial x \partial t}+\frac{\mathrm{d} c(t)}{\mathrm{d} t} \frac{\partial w}{\partial x}+c(t)^{2} \frac{\partial^{2} w}{\partial x^{2}}\right)+E I_{z} \frac{\partial^{4} w}{\partial x^{4}}-p \frac{\partial^{2} w}{\partial x^{2}}+\mu \frac{\partial w}{\partial t} \\
-E A\left[\frac{\partial u}{\partial x} \frac{\partial^{2} w}{\partial x^{2}}+\frac{\partial^{2} u}{\partial x^{2}} \frac{\partial w}{\partial x}+\frac{\partial w}{\partial x} \frac{\partial v}{\partial x} \frac{\partial^{2} v}{\partial x^{2}}+\frac{1}{2}\left(\frac{\partial v}{\partial x}\right)^{2} \frac{\partial^{2} w}{\partial x^{2}}+\frac{3}{2}\left(\frac{\partial w}{\partial x}\right)^{2} \frac{\partial^{2} w}{\partial x^{2}}\right]=0 \\
\rho A\left(\frac{\partial^{2} v}{\partial t^{2}}+2 c(t) \frac{\partial^{2} v}{\partial x \partial t}+\frac{\mathrm{d} c(t)}{\mathrm{d} t} \frac{\partial v}{\partial x}+c(t)^{2} \frac{\partial^{2} v}{\partial x^{2}}\right)+E I_{y} \frac{\partial^{4} v}{\partial x^{4}}-p \frac{\partial^{2} v}{\partial x^{2}}+\mu \frac{\partial v}{\partial t} \\
-E A\left[\frac{\partial u}{\partial x} \frac{\partial^{2} v}{\partial x^{2}}+\frac{\partial^{2} u}{\partial x^{2}} \frac{\partial v}{\partial x}+\frac{\partial v}{\partial x} \frac{\partial w}{\partial x} \frac{\partial^{2} w}{\partial x^{2}}+\frac{1}{2}\left(\frac{\partial w}{\partial x}\right)^{2} \frac{\partial^{2} v}{\partial x^{2}}+\frac{3}{2}\left(\frac{\partial v}{\partial x}\right)^{2} \frac{\partial^{2} v}{\partial x^{2}}\right]=0
\end{gathered}
$$

with the following boundary conditions for a hinged-hinged beam with immovable ends:

$$
\begin{gathered}
\left.u\right|_{x=0}=\left.u\right|_{x=L}=0 \\
\left.w\right|_{x=0}=\left.w\right|_{x=L}=0,\left.\quad \frac{\partial^{2} w}{\partial x^{2}}\right|_{x=0}=\left.\frac{\partial^{2} w}{\partial x^{2}}\right|_{x=L}=0
\end{gathered}
$$

$$
\left.v\right|_{x=0}=\left.v\right|_{x=L}=0,\left.\quad \frac{\partial^{2} v}{\partial x^{2}}\right|_{x=0}=\left.\frac{\partial^{2} v}{\partial x^{2}}\right|_{x=L}=0
$$

The dimensionless form of the equations of motion and boundary conditions is obtained by introducing the following dimensionless parameters: 


$$
\begin{aligned}
& u^{*}=\frac{u}{L}, \quad w^{*}=\frac{w}{L}, \quad v^{*}=\frac{v}{L}, \quad x^{*}=\frac{x}{L}, \quad t^{*}=t \sqrt{\frac{p}{\rho A L^{2}}}, \quad c_{0}^{*}=c_{0} \sqrt{\frac{\rho A}{p}}, \quad v_{f}=\sqrt{\frac{E I_{z}}{p L^{2}}}, \quad \Omega=\omega \sqrt{\frac{\rho A L^{2}}{p}}, \quad v_{1}=\sqrt{\frac{E A}{p}}, \\
& \alpha=\sqrt{\frac{I_{y}}{I_{z}}}, \quad \mu^{*}=\frac{\mu L}{p} \sqrt{\frac{p}{\rho A}} \quad c_{1}^{*}=c_{1} \sqrt{\frac{\rho A}{p}}
\end{aligned}
$$

substituting them into Eqs. (5)-(10) and disregarding the asterisk notation for briefness. This operation gives the following dimensionless nonlinear partial differential equations of motion for the longitudinal, transverse, and lateral motions, respectively:

$$
\frac{\partial^{2} u}{\partial t^{2}}+2 c(t) \frac{\partial^{2} u}{\partial x \partial t}+\frac{\mathrm{d} c(t)}{\mathrm{d} t}\left(1+\frac{\partial u}{\partial x}\right)+c(t)^{2} \frac{\partial^{2} u}{\partial x^{2}}-v_{1}^{2}\left(\frac{\partial^{2} u}{\partial x^{2}}+\frac{\partial v}{\partial x} \frac{\partial^{2} v}{\partial x^{2}}+\frac{\partial w}{\partial x} \frac{\partial^{2} w}{\partial x^{2}}\right)+\mu \frac{\partial u}{\partial t}=0
$$

(a)

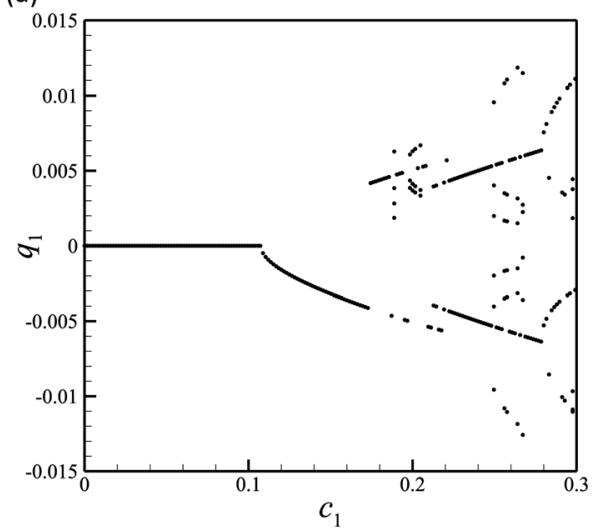

(c)

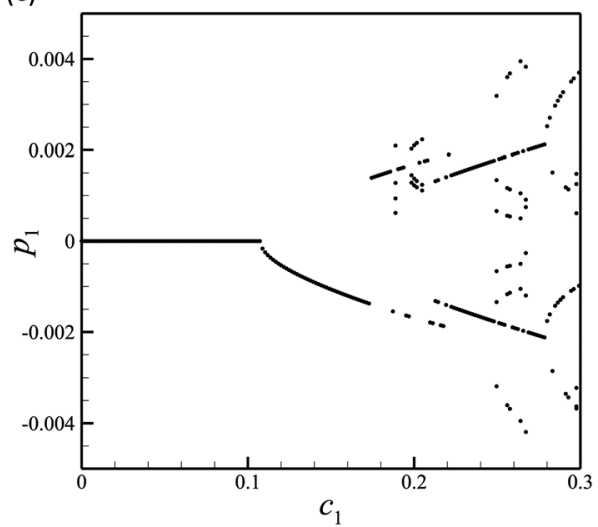

(e)

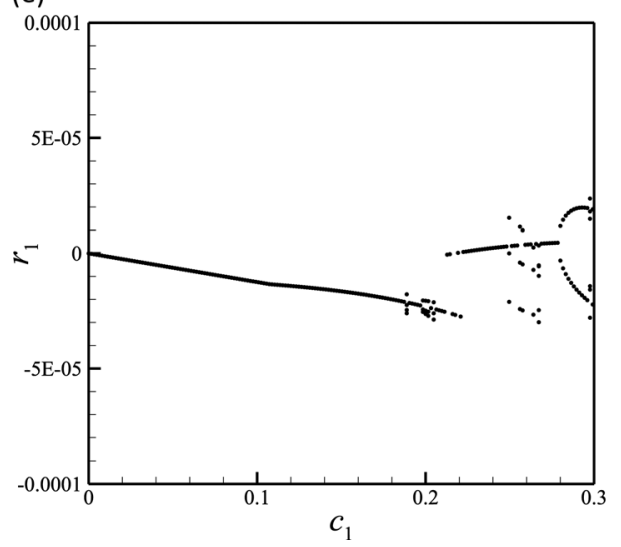

(b)

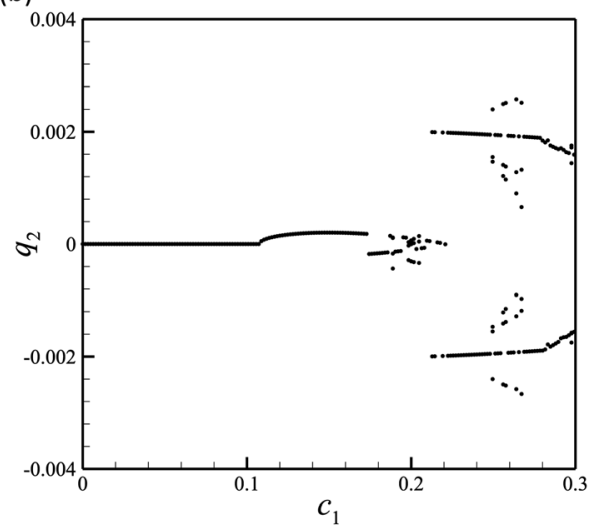

(d)

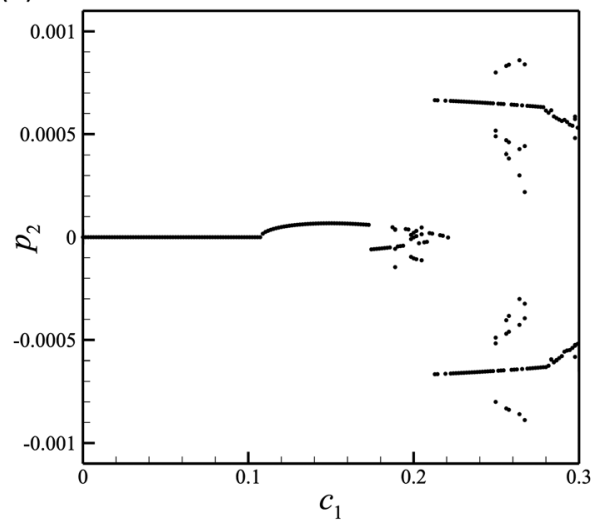

(f)

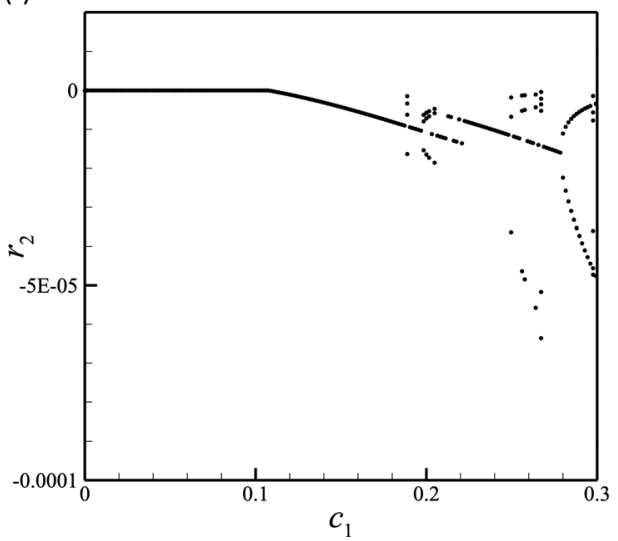

Fig. 2 Bifurcation diagrams of Poincaré points for increasing amplitude of the axial speed variations on the system with $c_{0}=1.00 ;((a)$ and $(b))$ the first two generalized coordinates of the transverse motion; $((c)$ and $(d))$ the first two generalized coordinates of the lateral motion; and $((e)$ and $(f))$ the first two generalized coordinates of the longitudinal motion 


$$
\begin{gathered}
\frac{\partial^{2} w}{\partial t^{2}}+2 c(t) \frac{\partial^{2} w}{\partial x \partial t}+\frac{\mathrm{d} c(t)}{\mathrm{d} t} \frac{\partial w}{\partial x}+\left(c(t)^{2}-1\right) \frac{\partial^{2} w}{\partial x^{2}}+v_{f}^{2} \frac{\partial^{4} w}{\partial x^{4}}+\mu \frac{\partial w}{\partial t} \\
-v_{1}^{2}\left[\frac{\partial u}{\partial x} \frac{\partial^{2} w}{\partial x^{2}}+\frac{\partial^{2} u}{\partial x^{2}} \frac{\partial w}{\partial x}+\frac{\partial w}{\partial x} \frac{\partial v}{\partial x} \frac{\partial^{2} v}{\partial x^{2}}+\frac{1}{2}\left(\frac{\partial v}{\partial x}\right)^{2} \frac{\partial^{2} w}{\partial x^{2}}+\frac{3}{2}\left(\frac{\partial w}{\partial x}\right)^{2} \frac{\partial^{2} w}{\partial x^{2}}\right]=0 \\
\frac{\partial^{2} v}{\partial t^{2}}+2 c(t) \frac{\partial^{2} v}{\partial x \partial t}+\frac{\mathrm{d} c(t)}{\mathrm{d} t} \frac{\partial v}{\partial x}+\left(c(t)^{2}-1\right) \frac{\partial^{2} v}{\partial x^{2}}+\alpha^{2} v_{f}^{2} \frac{\partial^{4} v}{\partial x^{4}}+\mu \frac{\partial v}{\partial t} \\
-v_{1}^{2}\left[\frac{\partial u}{\partial x} \frac{\partial^{2} v}{\partial x^{2}}+\frac{\partial^{2} u}{\partial x^{2}} \frac{\partial v}{\partial x}+\frac{\partial v}{\partial x} \frac{\partial w}{\partial x} \frac{\partial^{2} w}{\partial x^{2}}+\frac{1}{2}\left(\frac{\partial w}{\partial x}\right)^{2} \frac{\partial^{2} v}{\partial x^{2}}+\frac{3}{2}\left(\frac{\partial v}{\partial x}\right)^{2} \frac{\partial^{2} v}{\partial x^{2}}\right]=0
\end{gathered}
$$

(a)

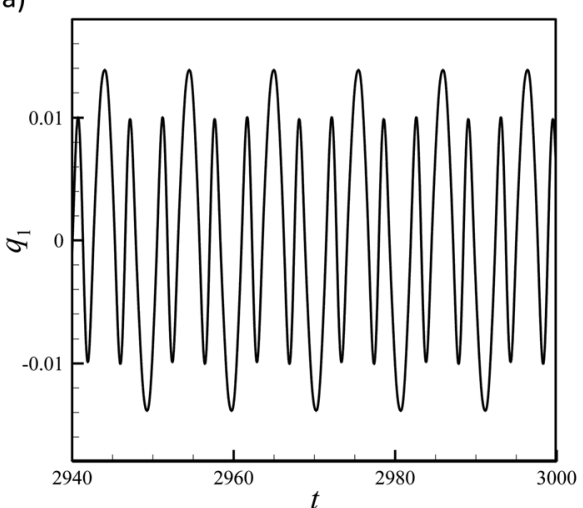

(c)

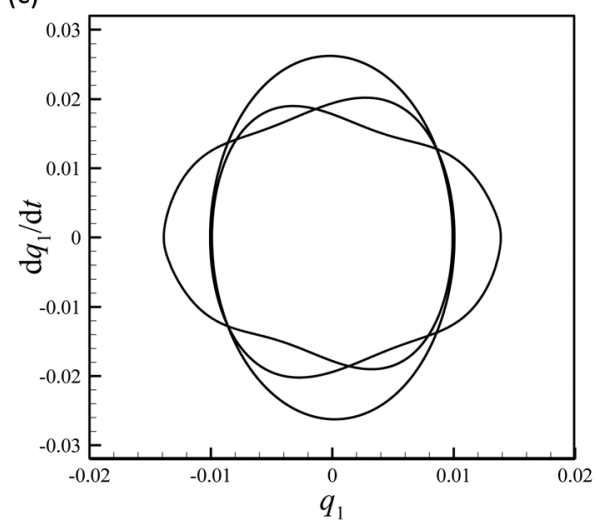

(e)

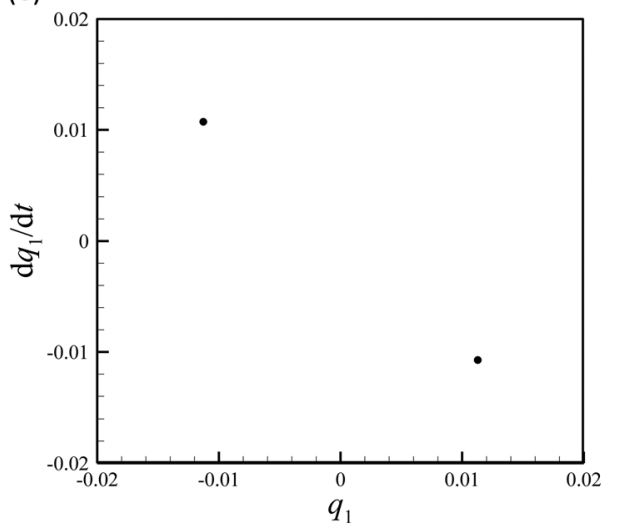

(b)

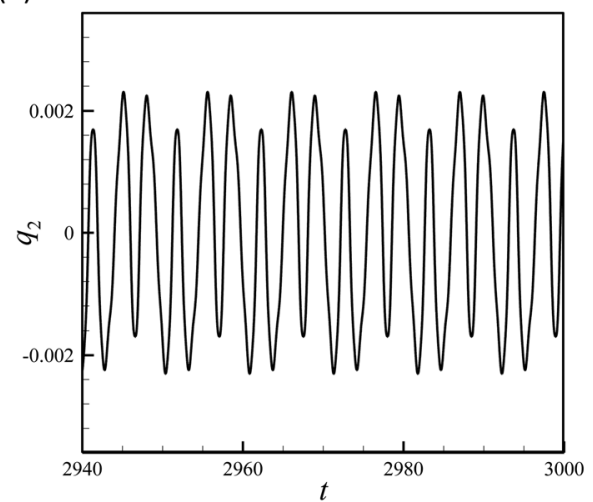

(d)

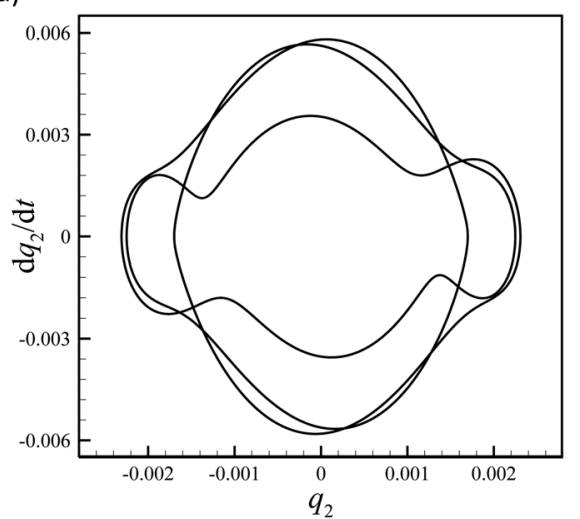

(f)

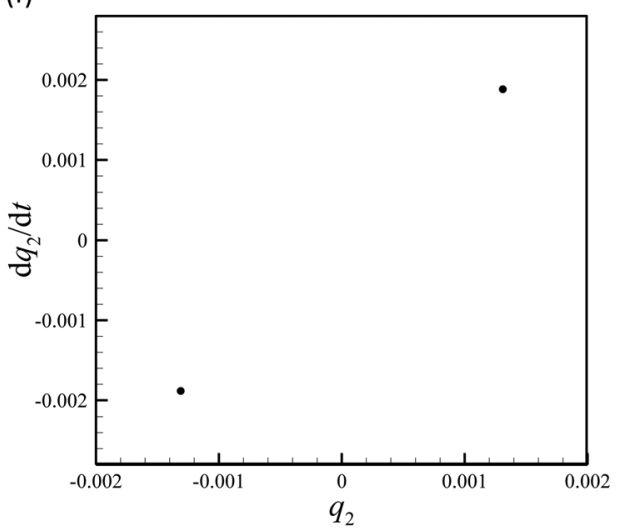

Fig. 3 Period-2 oscillations for the system of Fig. 2 at $c_{1}=0.2480$ : ((a) and $\left.(b)\right)$ time traces of the $q_{1}$ and $q_{2}$ motions, respectively; $((c)$ and $(d))$ phase-plane diagrams of the $q_{1}$ and $q_{2}$ motions, respectively; and $((e)$ and $(f))$ Poincare sections of the $q_{1}$ and $q_{2}$ motions, respectively 
with the following dimensionless boundary conditions:

$$
\begin{gathered}
\left.u\right|_{x=0}=\left.u\right|_{x=1}=0 \\
\left.w\right|_{x=0}=\left.w\right|_{x=1}=0,\left.\quad \frac{\partial^{2} w}{\partial x^{2}}\right|_{x=0}=\left.\frac{\partial^{2} w}{\partial x^{2}}\right|_{x=1}=0 \\
\left.v\right|_{x=0}=\left.v\right|_{x=1}=0,\left.\quad \frac{\partial^{2} v}{\partial x^{2}}\right|_{x=0}=\left.\frac{\partial^{2} v}{\partial x^{2}}\right|_{x=1}=0
\end{gathered}
$$

The presence of the time-dependent axial speed in the equations of motion (i.e., Eqs. (12)-(14)) renders the system parametrically excited gyroscopic. The class features include the generation of traveling wave components along the length of the beam as a result of gyroscopic forces and decreased flexural stiffness caused by the simultaneous presence of the axial speed and the deformation curvature of the beam. In what follows, the Galerkin method is employed to discretize Eqs. (12)-(14) into a set of second-order nonlinear ordinary differential equations; the eigenfunctions for the transverse motion of a hinged-hinged linear stationary beam are chosen as the appropriate basis functions for the motions in the $x, y$, and $z$ directions. The displacement field is approximated using the following series expansions: (a)

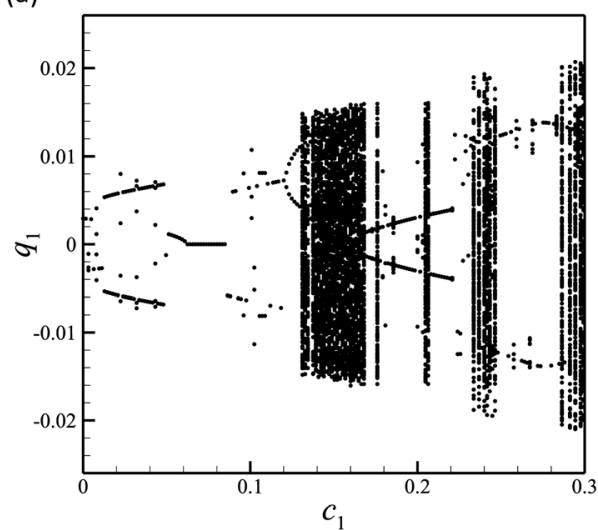

(c)

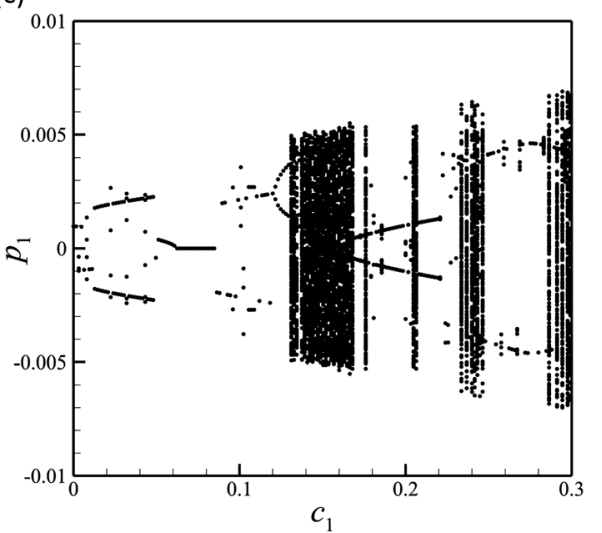

(e)

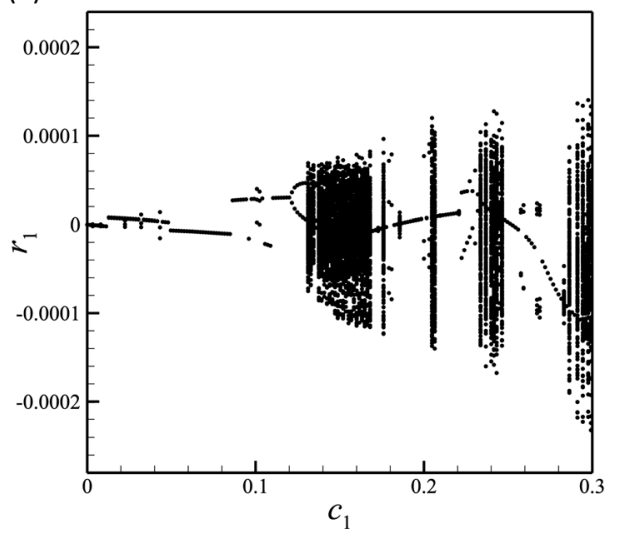

(b)

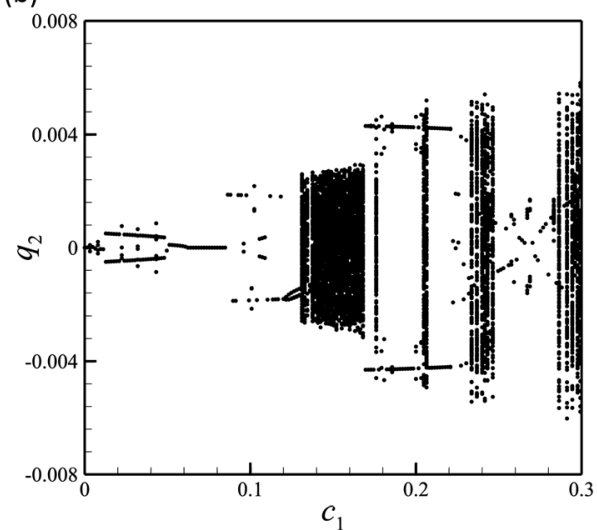

(d)

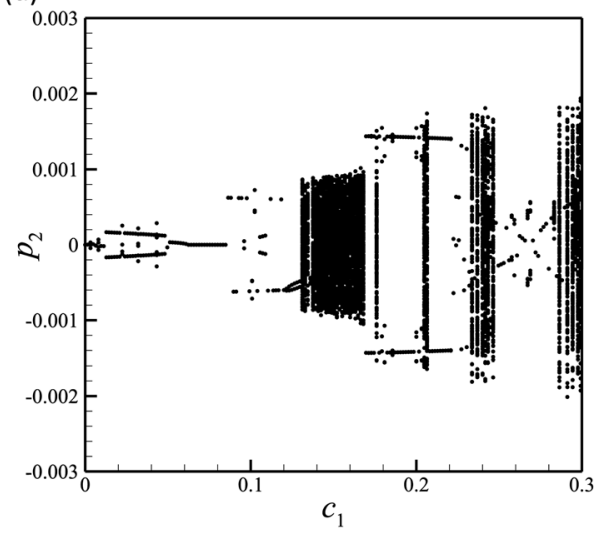

(f)

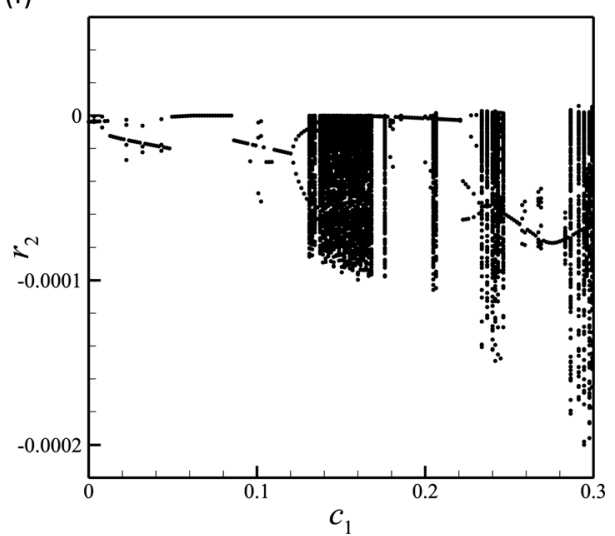

Fig. 4 Bifurcation diagrams of Poincaré points for increasing amplitude of the axial speed variations on the system with $c_{0}=1.15 ;((a)$ and $(b))$ the first two generalized coordinates of the transverse motion; $((c)$ and $(d))$ the first two generalized coordinates of the lateral motion; and $((e)$ and $(f))$ the first two generalized coordinates of the longitudinal motion 


$$
\begin{aligned}
& w(x, t)=\sum_{i=1}^{M} \phi_{i}(x) q_{i}(t) \\
& v(x, t)=\sum_{i=1}^{N} \phi_{i}(x) p_{i}(t) \\
& u(x, t)=\sum_{i=1}^{Q} \phi_{i}(x) r_{i}(t)
\end{aligned}
$$

(a)

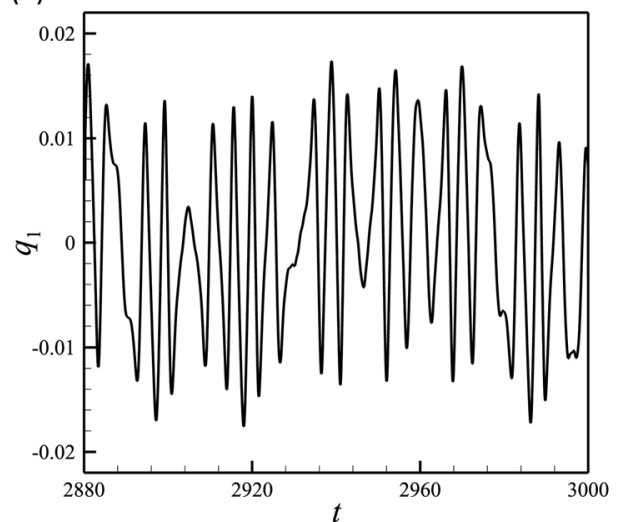

(c)

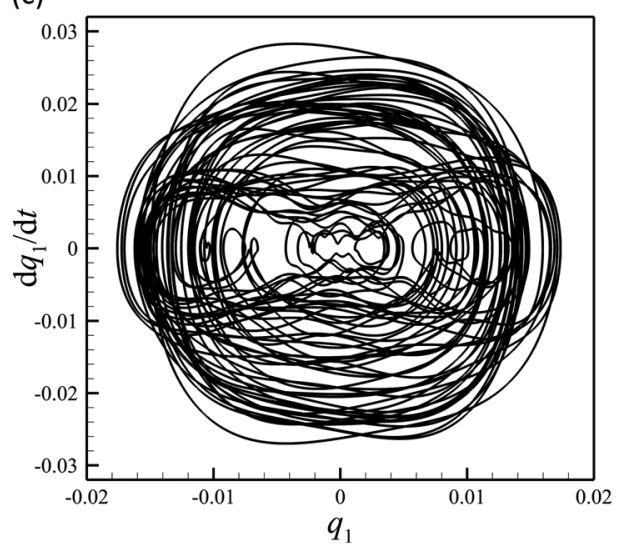

(e)

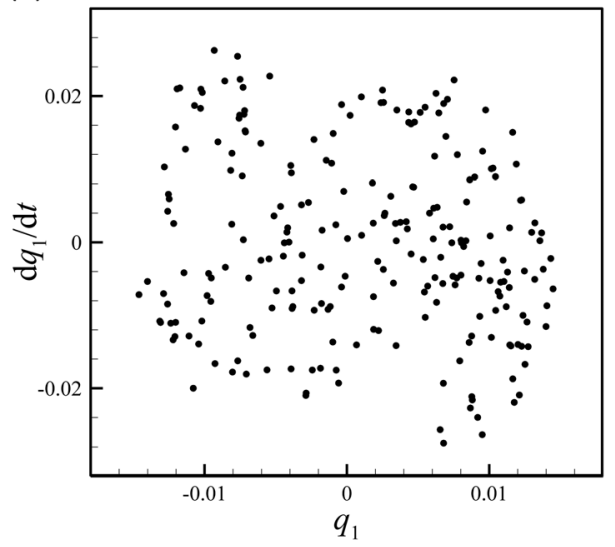

where $\phi_{i}(x)$ denotes the $i$ th eigenfunction for the transverse motion of a hinged-hinged linear stationary beam and $q_{i}(t)$, $p_{i}(t)$, and $r_{i}(t)$ denote the $i$ th generalized coordinates for the transverse, lateral, and longitudinal motions, respectively. The application of the Galerkin method states that Eqs. (18)-(20) are substituted into Eqs. (12)-(14) and the resultant equations are multiplied by the corresponding eigenfunction; integrating the final equations with respect to $x$ from 0 to 1 yields the following: (b)

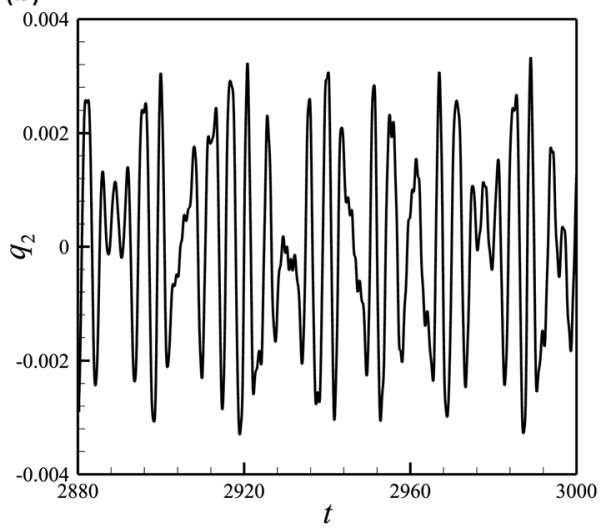

(d)

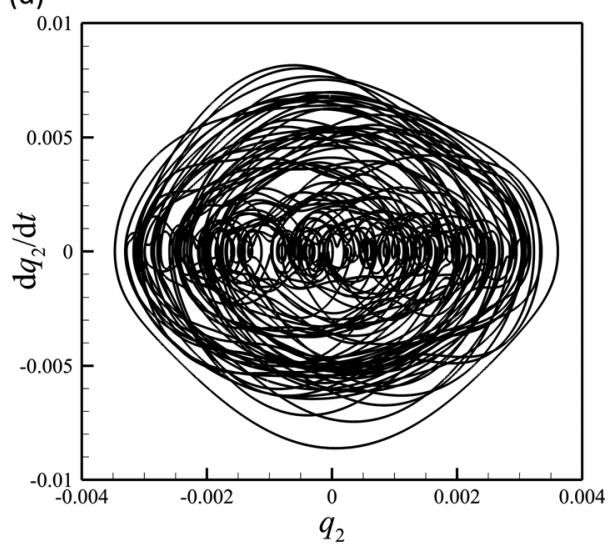

(f)

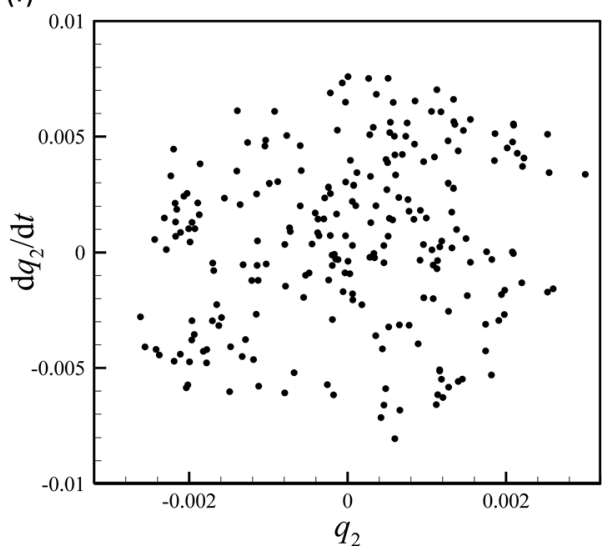

Fig. 5 Chaotic oscillations for the system of Fig. 4 at $c_{1}=0.1536$ : ((a) and $\left.(b)\right)$ time traces of the $q_{1}$ and $q_{2}$ motions, respectively; $((c)$ and $(d))$ phase-plane diagrams of the $q_{1}$ and $q_{2}$ motions, respectively; and $((e)$ and $(f))$ Poincare sections of the $q_{1}$ and $q_{2}$ motions, respectively 


$$
\begin{aligned}
& \sum_{j=1}^{Q}\left(\int_{0}^{1} \phi_{i} \phi_{j} \mathrm{~d} x\right) \ddot{r}_{j}+2\left(c_{0}+c_{1} \sin (\Omega t)\right) \sum_{j=1}^{Q}\left(\int_{0}^{1} \phi_{i} \phi_{j}^{\prime} \mathrm{d} x\right) \dot{r}_{j} \\
& \quad+c_{1} \Omega \cos (\Omega t)\left[\int_{0}^{1} \phi_{i} \mathrm{~d} x+\sum_{j=1}^{Q}\left(\int_{0}^{1} \phi_{i} \phi_{j}^{\prime} \mathrm{d} x\right) r_{j}\right]+\mu \sum_{j=1}^{Q}\left(\int_{0}^{1} \phi_{i} \phi_{j} \mathrm{~d} x\right) \dot{r}_{j} \\
& \quad+\left(c_{0}^{2}+c_{1}^{2}(\sin (\Omega t))^{2}+2 c_{0} c_{1} \sin (\Omega t)\right) \sum_{j=1}^{Q}\left(\int_{0}^{1} \phi_{i} \phi_{j}^{\prime \prime} \mathrm{d} x\right) r_{j} \\
& \quad-v_{1}^{2}\left[\sum_{j=1}^{Q}\left(\int_{0}^{1} \phi_{i} \phi_{j}^{\prime \prime} \mathrm{d} x\right) r_{j}+\sum_{j=1}^{N} \sum_{k=1}^{N}\left(\int_{0}^{1} \phi_{i} \phi_{j}^{\prime} \phi_{k}^{\prime \prime} \mathrm{d} x\right) p_{j} p_{k}+\sum_{j=1}^{M} \sum_{k=1}^{M}\left(\int_{0}^{1} \phi_{i} \phi_{j}^{\prime} \phi_{k}^{\prime \prime} \mathrm{d} x\right) q_{j} q_{k}\right]=0 \quad i=1,2, \ldots, Q
\end{aligned}
$$

(a)

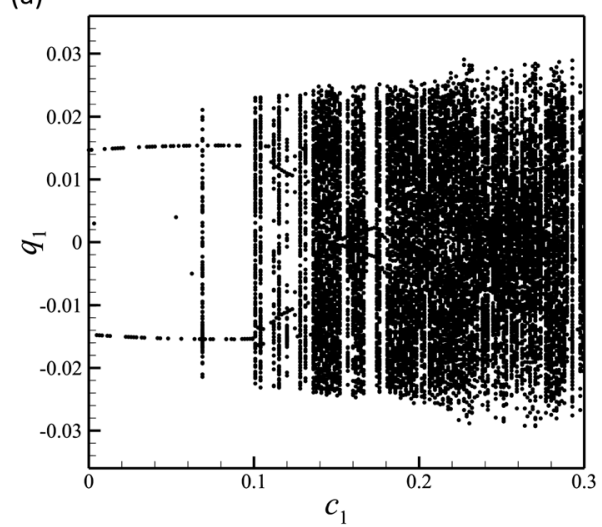

(c)

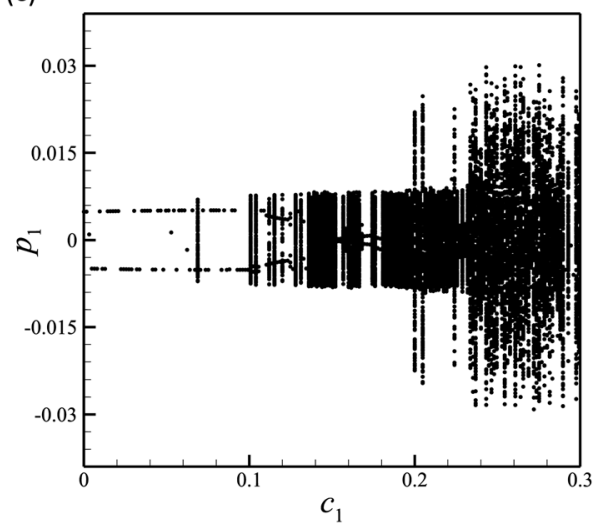

(e)

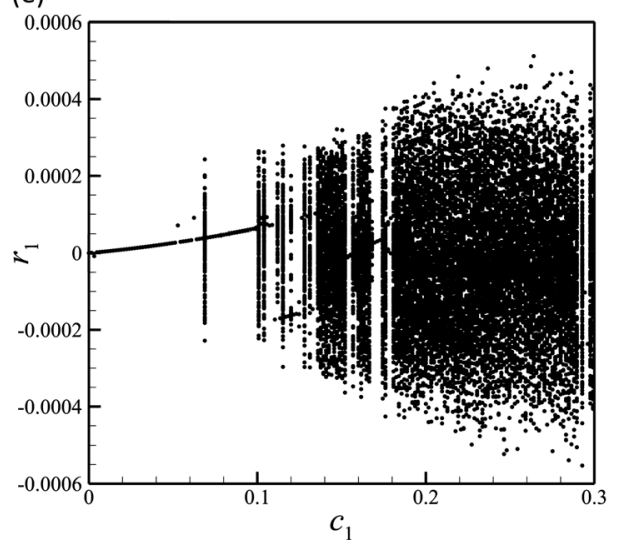

(b)

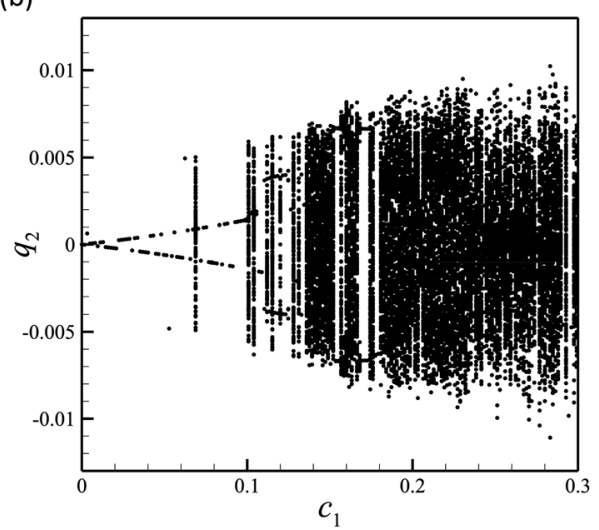

(d)

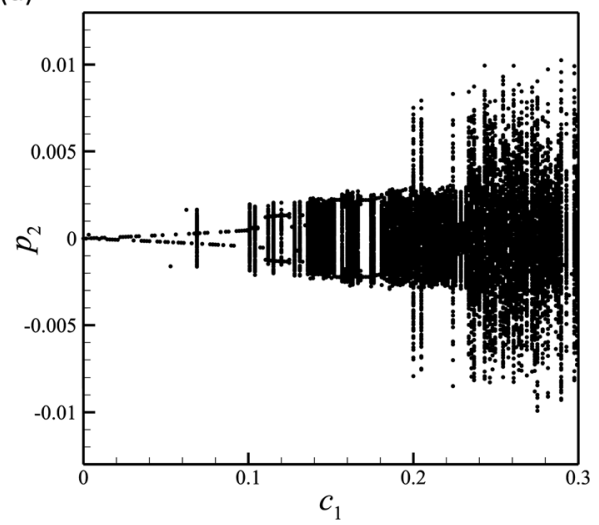

(f)

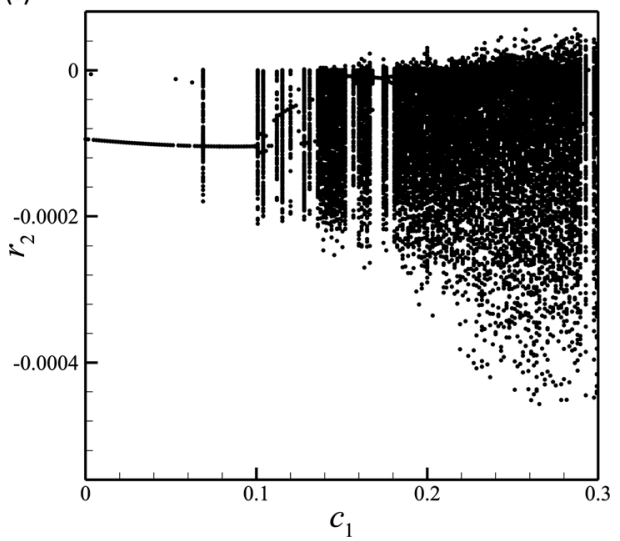

Fig. 6 Bifurcation diagrams of Poincaré points for increasing amplitude of the axial speed variations on the system with $c_{0}=1.40 ;((a)$ and $(b))$ the first two generalized coordinates of the transverse motion; $((c)$ and $(d))$ the first two generalized coordinates of the lateral motion; and $((e)$ and $(f))$ the first two generalized coordinates of the longitudinal motion 


$$
\begin{aligned}
& \sum_{j=1}^{M}\left(\int_{0}^{1} \phi_{i} \phi_{j} \mathrm{~d} x\right) \ddot{q}_{j}+2\left(c_{0}+c_{1} \sin (\Omega t)\right) \sum_{j=1}^{M}\left(\int_{0}^{1} \phi_{i} \phi_{j}^{\prime} \mathrm{d} x\right) \dot{q}_{j}+c_{1} \Omega \cos (\Omega t) \sum_{j=1}^{M}\left(\int_{0}^{1} \phi_{i} \phi_{j}^{\prime} \mathrm{d} x\right) q_{j} \\
& \quad+\left(c_{0}^{2}+c_{1}^{2}(\sin (\Omega t))^{2}+2 c_{0} c_{1} \sin (\Omega t)-1\right) \sum_{j=1}^{M}\left(\int_{0}^{1} \phi_{i} \phi_{j}^{\prime \prime} \mathrm{d} x\right) q_{j}+\mu \sum_{j=1}^{M}\left(\int_{0}^{1} \phi_{i} \phi_{j} \mathrm{~d} x\right) \dot{q}_{j} \\
& \quad+v_{f}^{2} \sum_{j=1}^{M}\left(\int_{0}^{1} \phi_{i} \phi_{j}^{\prime \prime \prime \prime} \mathrm{d} x\right) q_{j}-v_{1}^{2}\left[\sum_{j=1}^{Q} \sum_{k=1}^{M}\left(\int_{0}^{1} \phi_{i} \phi_{j}^{\prime} \phi_{k}^{\prime \prime} \mathrm{d} x\right) r_{j} q_{k}+\sum_{j=1}^{Q} \sum_{k=1}^{M}\left(\int_{0}^{1} \phi_{i} \phi_{j}^{\prime \prime} \phi_{k}^{\prime} \mathrm{d} x\right) r_{j} q_{k}\right. \\
& \quad+\sum_{j=1}^{M} \sum_{k=1}^{N} \sum_{l=1}^{N}\left(\int_{0}^{1} \phi_{i} \phi_{j}^{\prime} \phi_{k}^{\prime} \phi_{l}^{\prime \prime} \mathrm{d} x\right) q_{j} p_{k} p_{l}+\frac{1}{2} \sum_{j=1}^{N} \sum_{k=1}^{N} \sum_{l=1}^{M}\left(\int_{0}^{1} \phi_{i} \phi_{j}^{\prime} \phi_{k}^{\prime} \phi_{l}^{\prime \prime} \mathrm{d} x\right) p_{j} p_{k} q_{l} \\
& \left.\quad+\frac{3}{2} \sum_{j=1}^{M} \sum_{k=1}^{M} \sum_{l=1}^{M}\left(\int_{0}^{1} \phi_{i} \phi_{j}^{\prime} \phi_{k}^{\prime} \phi_{l}^{\prime \prime} \mathrm{d} x\right) q_{j} q_{k} q_{l}\right]=0, \quad i=1,2, \ldots, M
\end{aligned}
$$

(a)

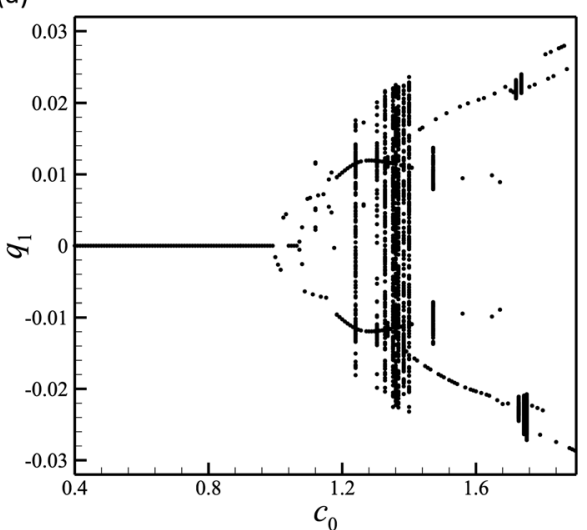

(c)

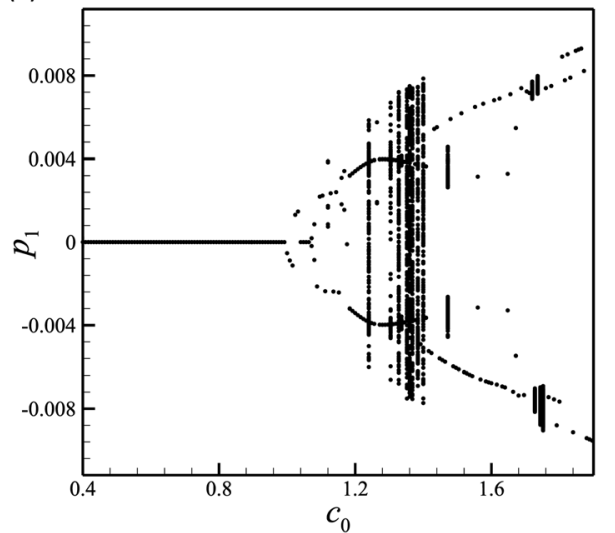

(e)

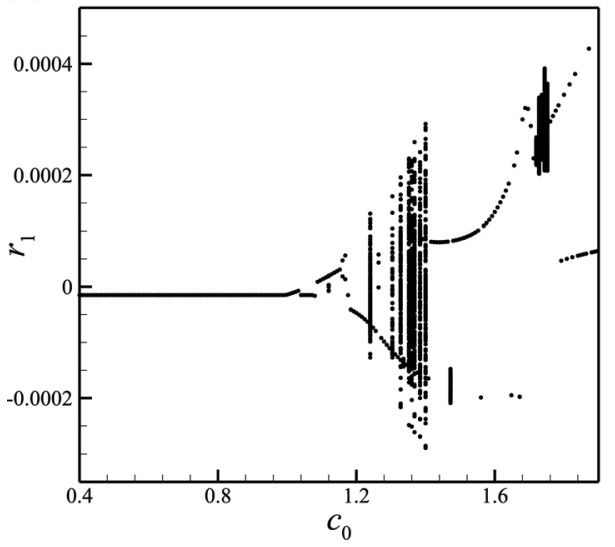

(b)

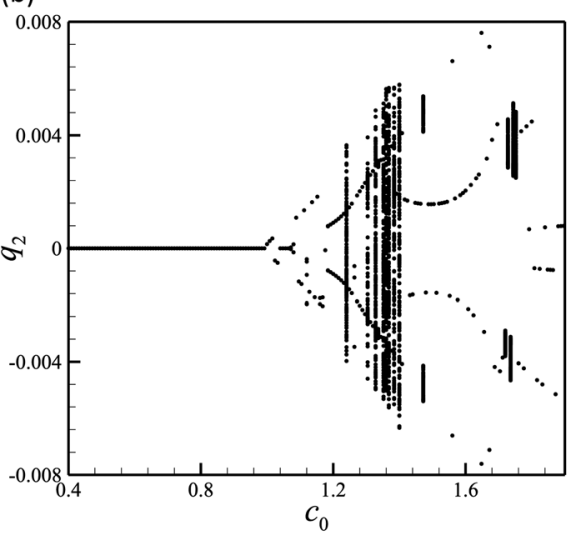

(d)

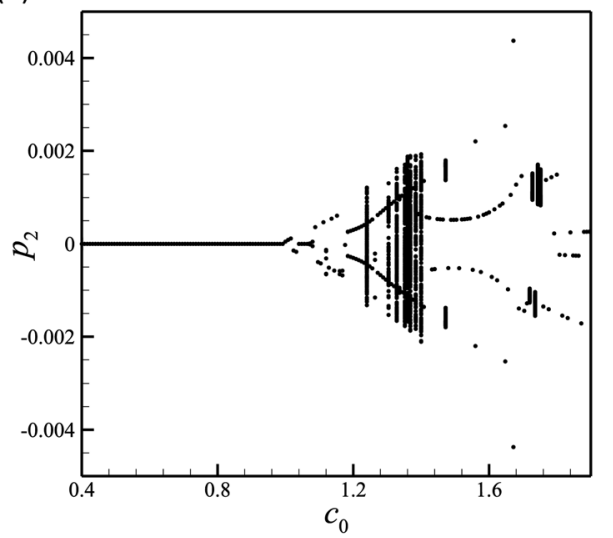

(f)

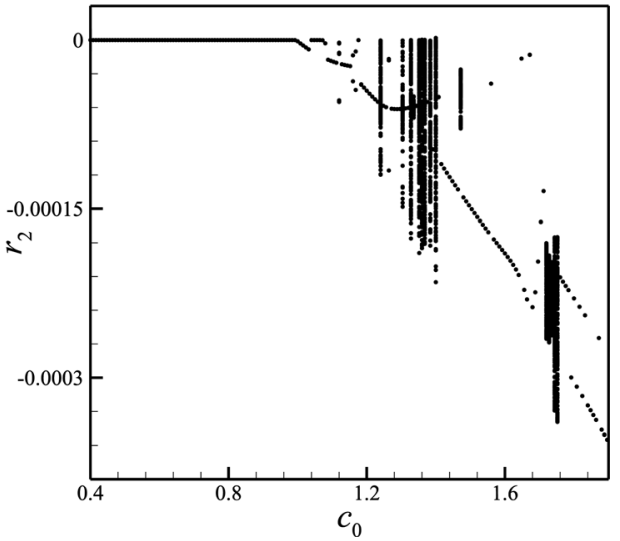

Fig. 7 Bifurcation diagrams of Poincaré points for increasing mean axial speed on the system with $c_{1}=0.12 ;((a)$ and $(b))$ the first two generalized coordinates of the transverse motion; $((c)$ and $(d))$ the first two generalized coordinates of the lateral motion; and $((e)$ and $(f))$ the first two generalized coordinates of the longitudinal motion 


$$
\begin{aligned}
& \sum_{j=1}^{N}\left(\int_{0}^{1} \phi_{i} \phi_{j} \mathrm{~d} x\right) \ddot{p}_{j}+2\left(c_{0}+c_{1} \sin (\Omega t)\right) \sum_{j=1}^{N}\left(\int_{0}^{1} \phi_{i} \phi_{j}^{\prime} \mathrm{d} x\right) \dot{p}_{j}+c_{1} \Omega \cos (\Omega t) \sum_{j=1}^{N}\left(\int_{0}^{1} \phi_{i} \phi_{j}^{\prime} \mathrm{d} x\right) p_{j} \\
& +\left(c_{0}^{2}+c_{1}^{2}(\sin (\Omega t))^{2}+2 c_{0} c_{1} \sin (\Omega t)-1\right) \sum_{j=1}^{N}\left(\int_{0}^{1} \phi_{i} \phi_{j}^{\prime \prime} \mathrm{d} x\right) p_{j}+\mu \sum_{j=1}^{N}\left(\int_{0}^{1} \phi_{i} \phi_{j} \mathrm{~d} x\right) \dot{p}_{j} \\
& \quad+\alpha^{2} v_{f}^{2} \sum_{j=1}^{N}\left(\int_{0}^{1} \phi_{i} \phi_{j}^{\prime \prime \prime \prime} \mathrm{d} x\right) p_{j}-v_{1}^{2}\left[\sum_{j=1}^{Q} \sum_{k=1}^{N}\left(\int_{0}^{1} \phi_{i} \phi_{j}^{\prime} \phi_{k}^{\prime \prime} \mathrm{d} x\right) r_{j} p_{k}+\sum_{j=1}^{Q} \sum_{k=1}^{N}\left(\int_{0}^{1} \phi_{i} \phi_{j}^{\prime \prime} \phi_{k}^{\prime} \mathrm{d} x\right) r_{j} p_{k}\right. \\
& \quad+\sum_{j=1}^{N} \sum_{k=1}^{M} \sum_{l=1}^{M}\left(\int_{0}^{1} \phi_{i} \phi_{j}^{\prime} \phi_{k}^{\prime} \phi_{l}^{\prime \prime} \mathrm{d} x\right) p_{j} q_{k} q_{l}+\frac{1}{2} \sum_{j=1}^{M} \sum_{k=1}^{M} \sum_{l=1}^{N}\left(\int_{0}^{1} \phi_{i} \phi_{j}^{\prime} \phi_{k}^{\prime} \phi_{l}^{\prime \prime} \mathrm{d} x\right) q_{j} q_{k} p_{l} \\
& \left.\quad+\frac{3}{2} \sum_{j=1}^{N} \sum_{k=1}^{N} \sum_{l=1}^{N}\left(\int_{0}^{1} \phi_{i} \phi_{j}^{\prime} \phi_{k}^{\prime} \phi_{l}^{\prime \prime} \mathrm{d} x\right) p_{j} p_{k} p_{l}\right]=0, \quad i=1,2, \ldots, N
\end{aligned}
$$

Equations (21)-(23) form a set of $(M+N+Q)$ second-order nonlinear ordinary differential equations with coupled terms and time-dependent coefficients; the dot and prime notations represent the differentiations with respect to the dimensionless time and axial coordinate, respectively. These equations are transformed into a new set of $2(M+N+Q)$ first-order nonlinear ordinary differential equations through use of a change of variables via $x_{i}=\dot{q}_{i}, y_{i}=\dot{p}_{i}$, and $z_{i}=\dot{r}_{i}$. In the present study, $M=N=7$ and $Q=10$ are chosen (i.e., a 24-degree-of-freedom system resulting in 48 first-order nonlinear ordinary differential equations with coupled nonlinear terms is employed); this number of modes ensures reliable results.

The time histories of the generalized coordinates are obtained by conducting direct time integration upon the discretized equations of motion. Sectioning these time traces in every period of the amplitude of the speed variations results in the Poincaré maps of the system. The Poincaré maps are plotted together by varying the mean axial speed or the amplitude of the speed variation as the bifurcation parameter, yielding the bifurcation diagrams of Poincaré maps of the system. Phase-plane diagrams and FFTs are also plotted using the time traces.

\section{Bifurcation Diagrams of Poincaré Maps}

The nonlinear global dynamical behavior of the system is examined numerically via conducting time integration upon the discretized equations of motion through use of the variable stepsize Runge-Kutta method, resulting in the time-varying generalized coordinates. The time integration was performed for a (a)

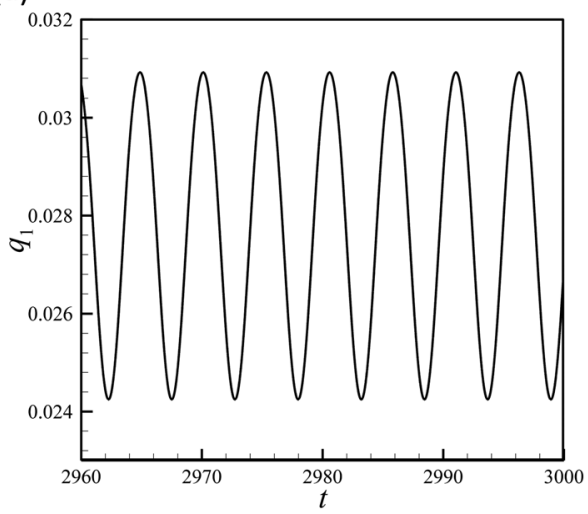

(c)

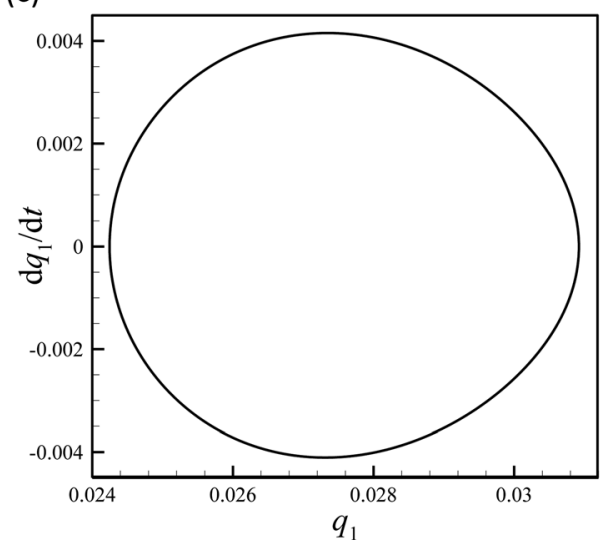

(b)

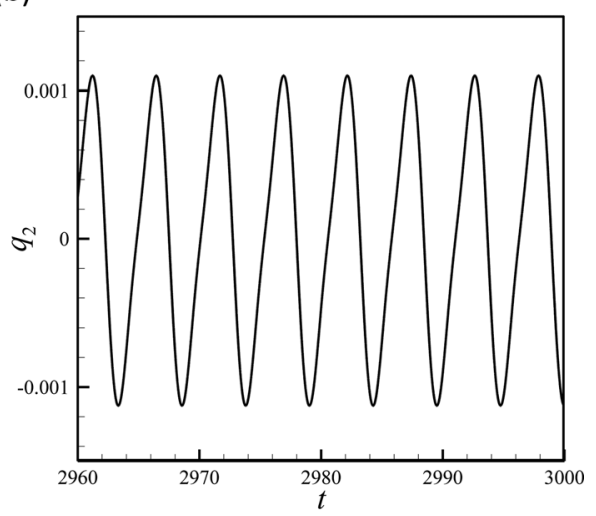

(d)

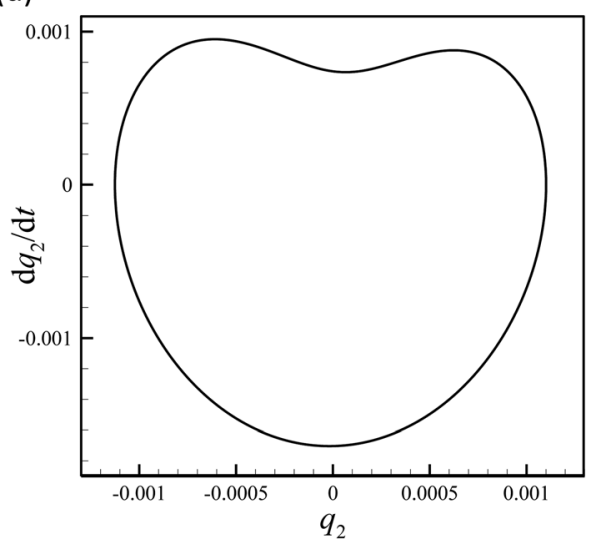

Fig. 8 Periodic oscillations for the system of Fig. 7 at $c_{0}=1.856:((a)$ and $(b))$ time traces of the $q_{1}$ and $q_{2}$ motions, respectively; and $((c)$ and $(d))$ phase-plane diagrams of the $q_{1}$ and $q_{2}$ motions, respectively 
sufficient time interval of [0 3000] dimensionless seconds; in order to exclude any possible transient effects and to make sure that the true dynamics is detected, only the last $30 \%$ of the response was retained. The bifurcation diagrams of Poincaré maps were then constructed by sectioning the time-varying generalized coordinates in every period of the frequency of the axial speed variations, as the amplitude of the axial speed variations or mean axial speed is varied as the bifurcation parameter. The excitation frequency and $\alpha$ are chosen as 1.2 and 1, respectively, for all the cases presented in this section. It should be noted that considering a circular cross section $(\alpha=1)$ results in a system with axisymmetric configuration with respect to the centerline of the beam, yielding the same linear natural frequencies for the transverse and lateral motions; in other words, the system possesses one-to-one internal resonances. In this context, the response and amplitude are referred to the $q_{1}$ motion and the amplitude of the $q_{1}$ motion where it is sectioned, respectively.

3.1 Amplitude of the Axial Speed Variations as the Bifurcation Parameter. The bifurcation diagrams of Poincaré maps are constructed by varying the amplitude of the axial speed variations, $c_{1}$, as the bifurcation parameter. Three different values of the mean axial speed are chosen, namely, $c_{0}=1.00,1.15$, and 1.40 , and the corresponding bifurcation diagrams are plotted in Figs. 2, 4, and 6, respectively. The following dimensionless system parameters are selected for the numerical calculations of this section: $v_{1}=33.5261, v_{f}=0.1732, \alpha=1, \mu=0.04$, and $\Omega=1.2$. (a)

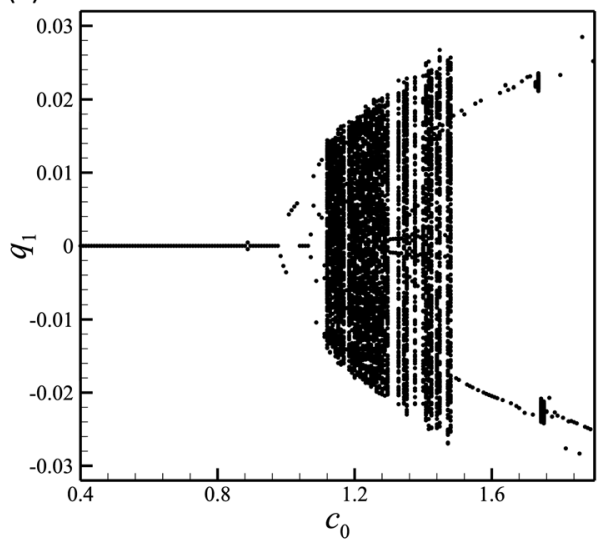

(c)

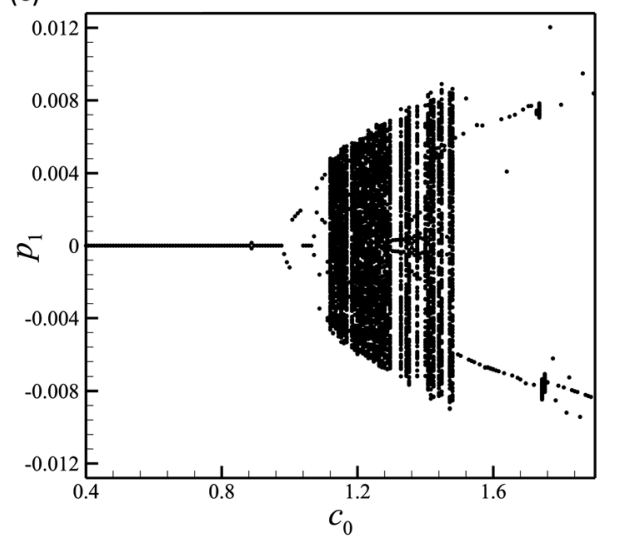

(e)

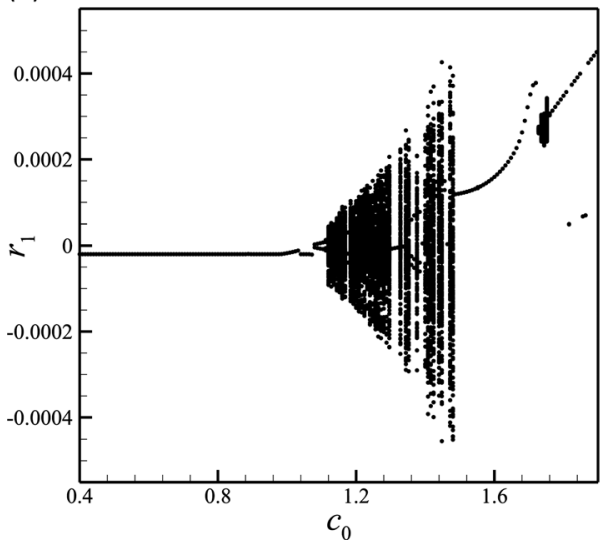

(b)

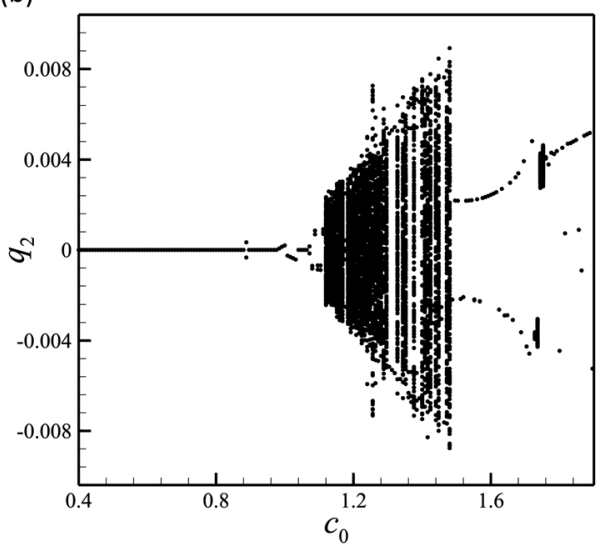

(d)

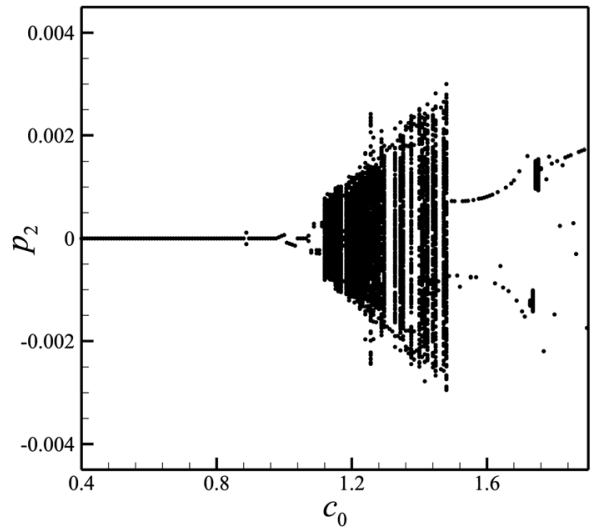

(f)

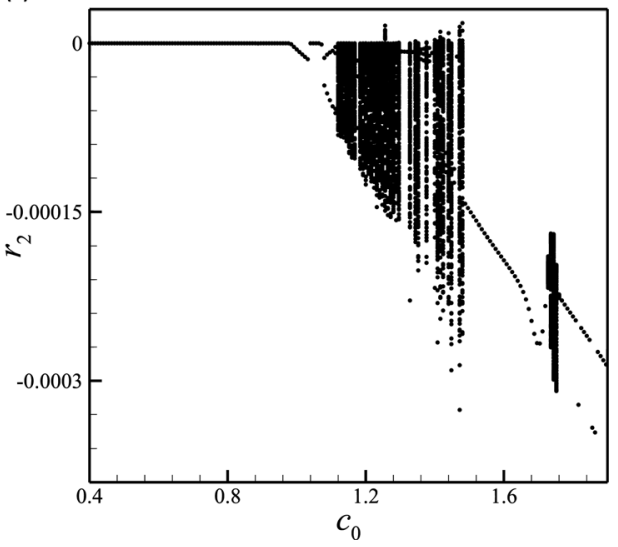

Fig. 9 Bifurcation diagrams of Poincaré points for increasing mean axial speed on the system with $c_{1}=0.16$; $((a)$ and $(b))$ the first two generalized coordinates of the transverse motion; $((c)$ and $(d))$ the first two generalized coordinates of the lateral motion; and $((e)$ and $(f))$ the first two generalized coordinates of the longitudinal motion 
Figure 2 illustrates the bifurcation diagrams of the system with $c_{0}=1.00$; as shown in the figure, after a range of zero response, the first periodic attractor is displayed by the system at $c_{1}=0.1088$. The amplitude of this periodic attractor increases with increasing bifurcation parameter $\left(c_{1}\right)$ until $c_{1}=0.1744$ is reached, where a saddle-node bifurcation occurs which appears as a jump in the bifurcation diagram. As the amplitude of the axial speed variations is increased further, the system displays different types of attractors, such as periodic, period-3, and period-4 until $c_{1}=0.2118$ is hit, where the motion becomes period- 2 ; this period-2 attractor prevails in the range of [0.2128 0.3000] with short bursts of period- 6 motion in between. The details of a period-2 attractor at $c_{1}=0.2480$ are plotted in Fig. 3 through time histories, phase-plane portraits, and Poincaré sections.

Increasing the mean axial speed to $c_{0}=1.15$, form $c_{0}=1.00$ in the system of Fig. 2, a new set of bifurcation diagrams is plotted

(a)

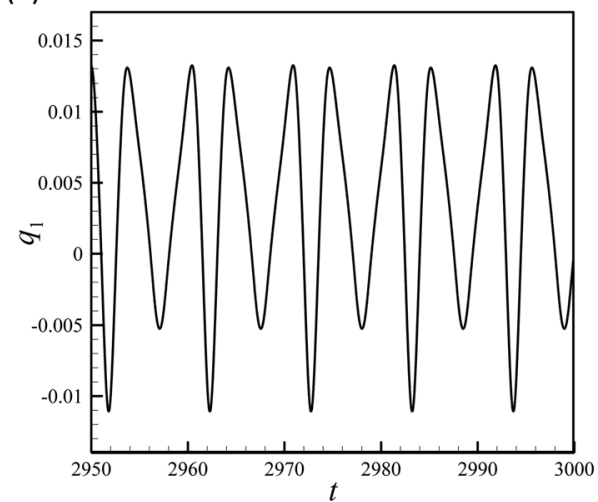

(c)

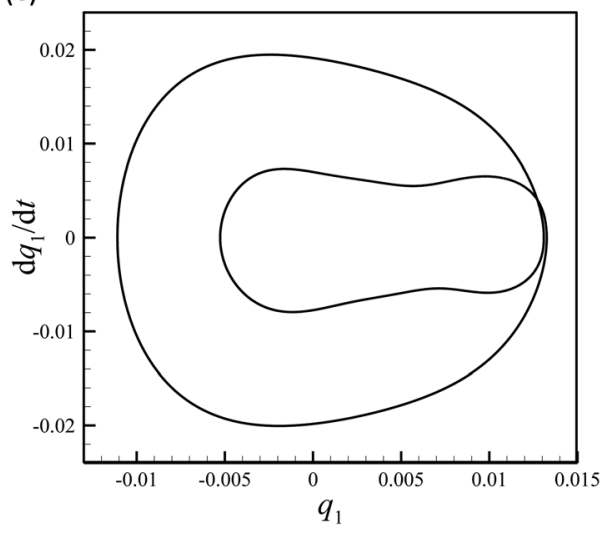

(e)

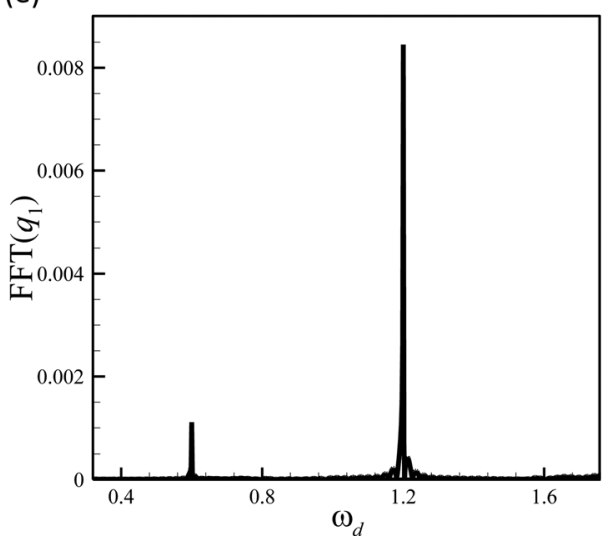

in Fig. 4. As shown in Fig. 4(a), the number of chaotic regions increases substantially as a result of increased mean axial speed. Specifically, as the bifurcation parameter is increased, a dominant period-2 motion along with other attractors such as period-3, period-4, and period- 6 is observed until $c_{1}=0.0496$ is reached; at this point, the dynamical system regains the periodic motion. By further increasing the bifurcation parameter, the amplitude of the response decreases up to $c_{1}=0.0624$, where the amplitude becomes zero. After a range of zero amplitude, the system displays periodic oscillations once again at $c_{1}=0.0864$. A period-3 attractor occurs at $c_{1}=0.1008$, which is very short-lived and by a small increment in the amplitude of the axial speed variations the dynamical system regains the original period at $c_{1}=0.1040$. Beyond that point, a period- 2 motion prevails until $c_{1}=0.1312$ is hit, where a direct transition from a period-2 to a chaotic motion is observed. The aforementioned period- 2 and chaotic attractors

(b)

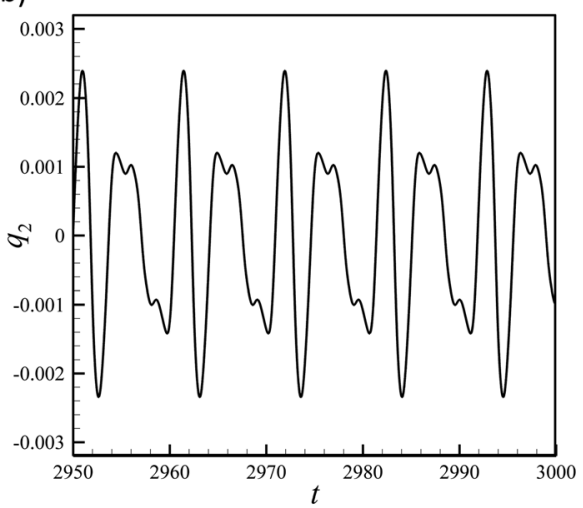

(d)

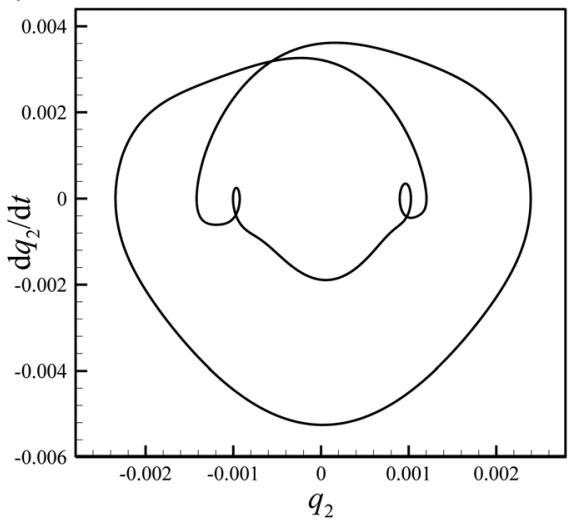

(f)

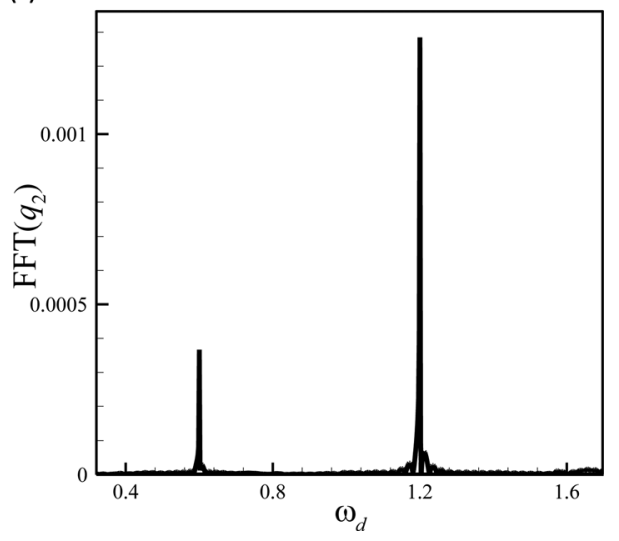

Fig. 10 Period-2 oscillations for the system of Fig. 9 at $c_{0}=1.104$ : $((a)$ and $(b))$ time traces of the $q_{1}$ and $q_{2}$ motions, respectively; $((c)$ and $(d))$ phase-plane diagrams of the $q_{1}$ and $q_{2}$ motions, respectively; and $((e)$ and $(f))$ FFTs of the $q_{1}$ and $q_{2}$ motions, respectively. $\omega_{d}$ is the dimensionless frequency. 
coexist in the rage of [0.1312 0.2208], while first the chaotic motion prevails until $c_{1}=0.1696$ is hit; the motion is characterized dominantly by period-2 attractor afterward up to $c_{1}=0.2208$. Beyond that point, there are different chaotic regions connected together by various attractors, such as periodic, period-2, period3 , and period- 4 . The characteristics of the chaotic attractor at $c_{1}=0.1536$ are illustrated in Fig. 5 .

The last set of bifurcation diagrams in this section corresponds to the system with $c_{0}=1.40$; this value of the mean axial speed is higher than previous cases. It is interesting noting that the motion amplitude is a nonzero value for $c_{1}=0$, implying that the system is in the supercritical regime and the oscillations (for nonzero values of $c_{1}$ ) occur over the buckled state. As shown in the figure, two limit cycles coexist as attractors of the system in the range of
[0.000 0.0992], where the final state of the system jumps from one to the other. Beyond that the motion is dominantly chaotic until $c_{1}=0.3000$ is reached. Comparing Figs. 2, 4, and 6, one can draw the general conclusion that, due to increased mean axial speed, the number of chaotic regions increases and chaos regions become wider; moreover, the number of higher-order bifurcation decreases.

3.2 Mean Axial Speed as the Bifurcation Parameter. In this section, the mean axial speed, $c_{0}$, is varied as the bifurcation parameter. The bifurcation diagrams of the system are constructed for different values of the amplitude of the axial speed variations, namely, $c_{1}=0.12,0.16,0.20$, and 0.4 , and plotted in (a)

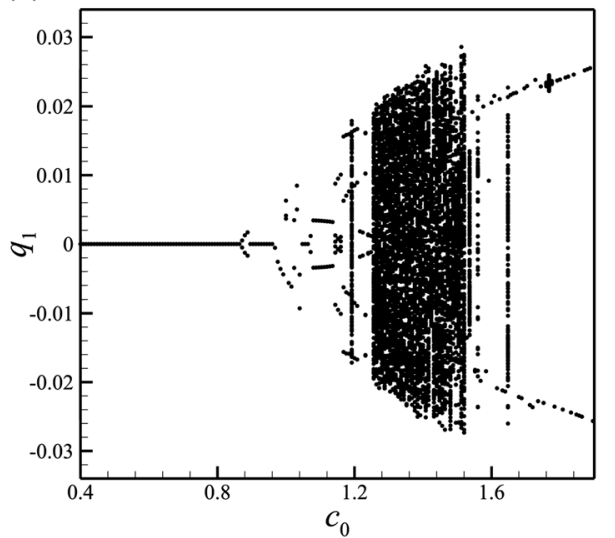

(c)

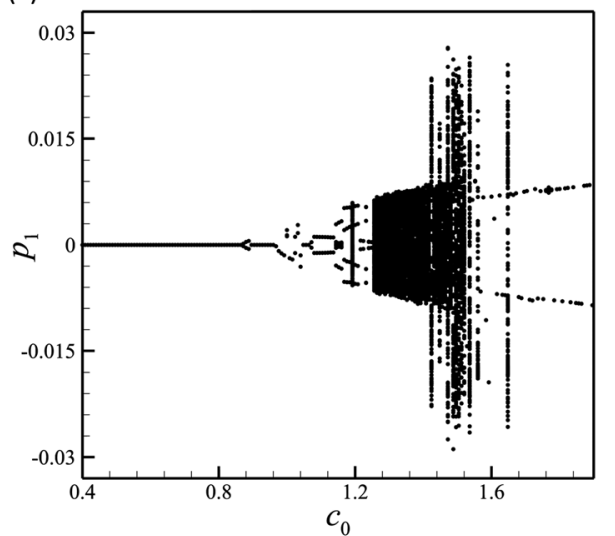

(e)

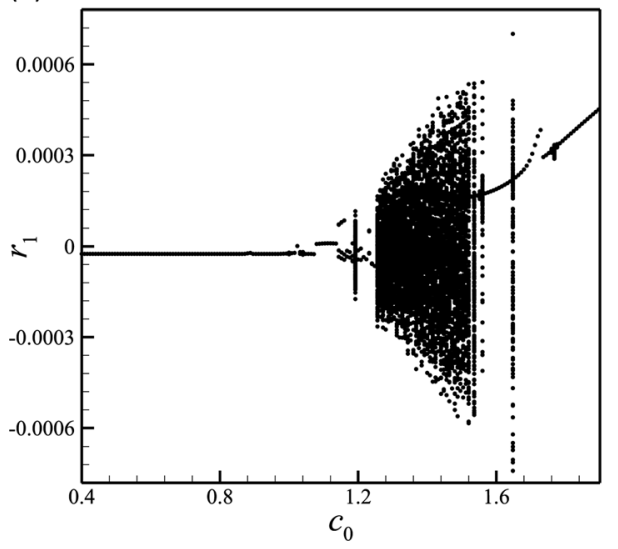

(b)

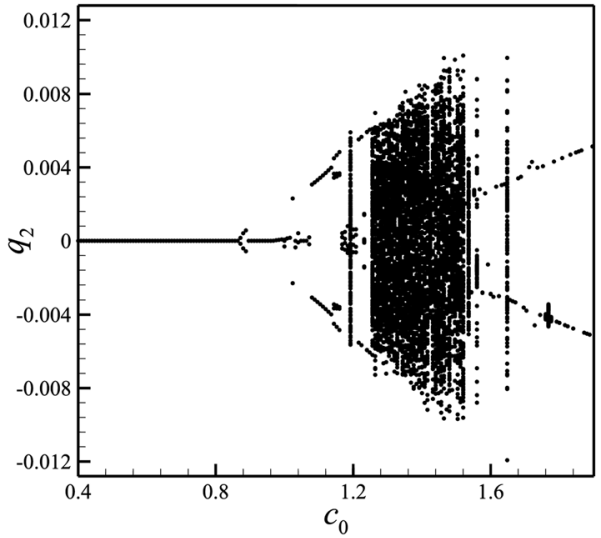

(d)

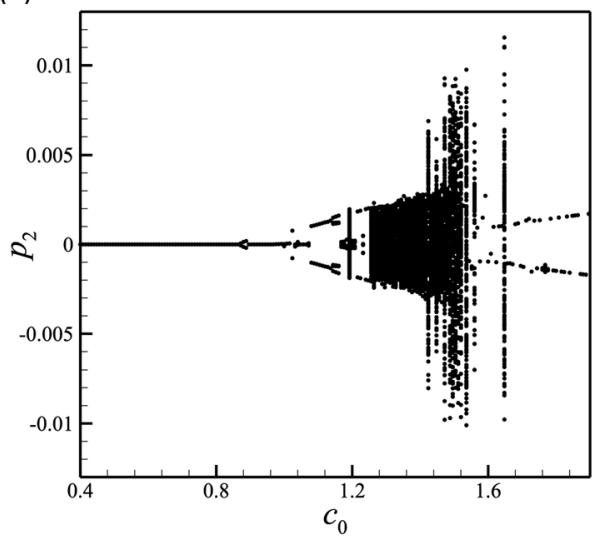

(f)

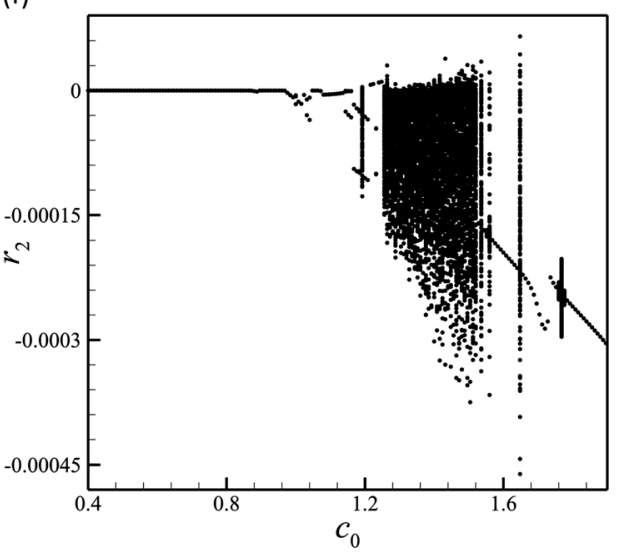

Fig. 11 Bifurcation diagrams of Poincaré points for increasing mean axial speed on the system with $c_{1}=0.20 ;((a)$ and $(b))$ the first two generalized coordinates of the transverse motion; $((c)$ and $(d))$ the first two generalized coordinates of the lateral motion; and $((e)$ and $(f))$ the first two generalized coordinates of the longitudinal motion 
Figs. 7, 9, 11, and 13, respectively. The numerical calculations of this section are performed by selecting the following dimensionless system parameters: $v_{1}=33.5261, v_{f}=0.1732, \alpha=1$, $\mu=0.04$, and $\Omega=1.2$.

Figure 7 shows the bifurcation diagrams of Poincaré maps of the system with $c_{1}=0.12$. Figure $7(a)$ illustrates that as the mean axial speed is increased, the system displays zero response until $c_{0}=1.000$ is reached where a jump occurs in the bifurcation diagram. A short burst of a period-2 motion is displayed by the system in the mean axial speed range of [1.072 1.080], continued by pure periodic oscillations. There are two coexisting periodic attractors in the interval [1.088 1.152], where the response repeatedly jumps from one to the other. By further increasing the mean axial speed, a period-2 motion prevails until $c_{0}=1.24$ is hit, where it starts coexisting with a chaotic motion; these two coexisting attractors can be seen in the mean axial speed range of [1.240 1.400]. By further incrementing the mean axial speed, the motion is dominantly characterized by two limit cycles, which coexist as periodic attractors and the final state of the system jumps from one to the other, until $c_{0}=1.900$; there are also a number of quasi-periodic and period-2 attractors in between. The characteristics of a periodic motion at $c_{0}=1.856$ are illustrated in Fig. 8 via time traces and phase-plane portraits of the first two generalized coordinates of the transverse motion.

A new set of bifurcation diagrams is constructed and plotted in Fig. 9 by increasing the amplitude of the axial speed variation (a)

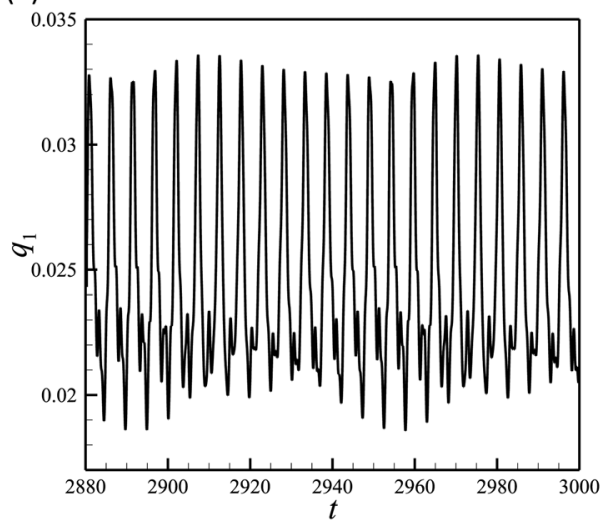

(c)

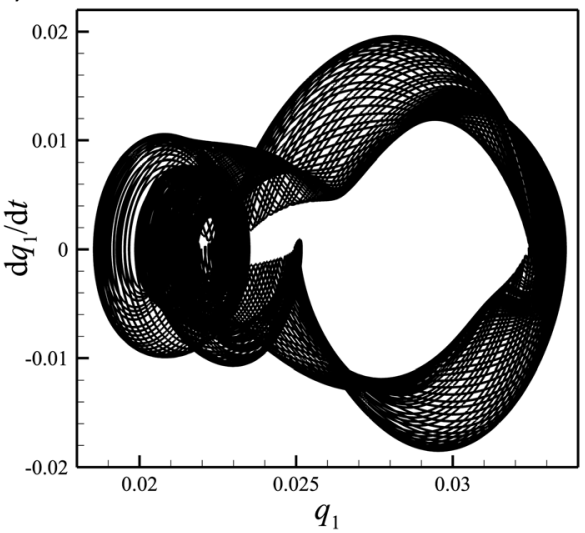

(e)

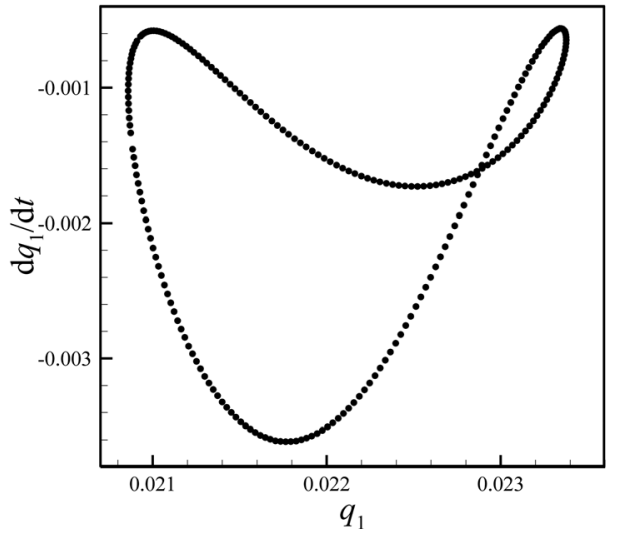

(b)

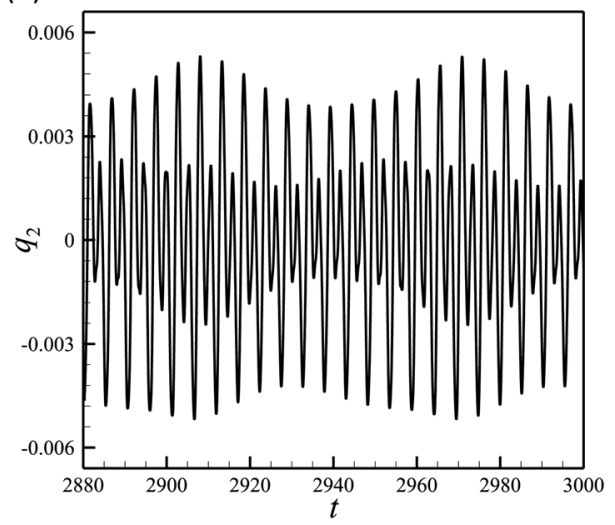

(d)

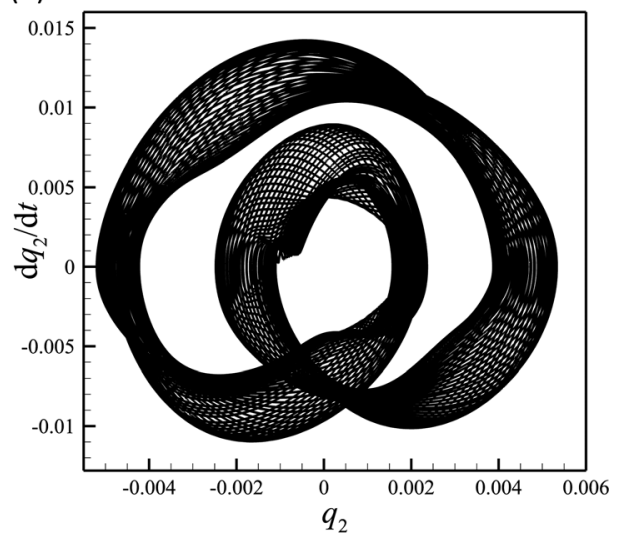

(f)

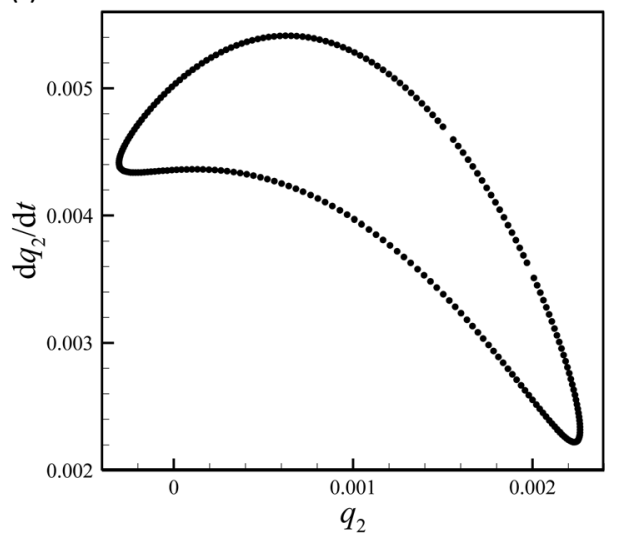

Fig. 12 Quasi-periodic oscillations for the system of Fig. 11 at $c_{0}=1.768$ : $((a)$ and $(b))$ time traces of the $q_{1}$ and $q_{2}$ motions, respectively; $((c)$ and $(d))$ phase-plane diagrams of the $q_{1}$ and $q_{2}$ motions, respectively; and $((e)$ and $(f))$ Poincaré sections of the $q_{1}$ and $q_{2}$ motions, respectively 
from $c_{1}=0.12$, in Fig. 7 , to $c_{1}=0.16$. As shown in the figure, the zero response is observed until $c_{0}=0.888$ is hit; at this point, a period-2 motion occurs which is very short-lived and by a small increment in $c_{0}$ the system returns to the zero amplitude motion at $c_{0}=0.896$. By further incrementing the mean axial speed, the first jump occurs at $c_{0}=0.984$, which is a lower value compared to the system of Fig. 7. The system displays pure periodic oscillations with different jumps up to $c_{0}=1.072$ where the attractor is changed to a period- 2 type; this type of motion is displayed by the system in the interval [1.072 1.104]. As the mean axial speed is increased further, a direct transition from a period- 2 motion to a chaotic one occurs at $c_{0}=1.120$; this chaotic attractor prevails in the range of $\left[\begin{array}{lll}1.120 & 1.480\end{array}\right]$ with windows of period-2, period-3, and period- 6 motions in between. Beyond that the motion is characterized dominantly by the two coexisting periodic attractors until $c_{0}=1.900$ is reached. Figure 10 shows the typical characteristics of a period-2 attractor at $c_{0}=1.104$.

The bifurcation diagrams for higher values of the amplitude of the axial speed variations, i.e., $c_{1}=0.20$ and 0.40 are depicted in Figs. 11 and 13, respectively. Comparing Figs. 11, and 13, one can draw the general conclusion that, due to increased amplitude of the axial speed variations, the number of higher-order bifurcations decreases and the first bifurcation occurs at lower mean axial speeds. Moreover, it is concluded that the chaotic attractors become stronger and the chaotic regions become wider. The overall amplitude of the oscillations is also increased, as a result of increasing the amplitude of the axial speed variations. The details of a quasi-periodic and a chaotic attractor are depicted in Figs. 12

(b)

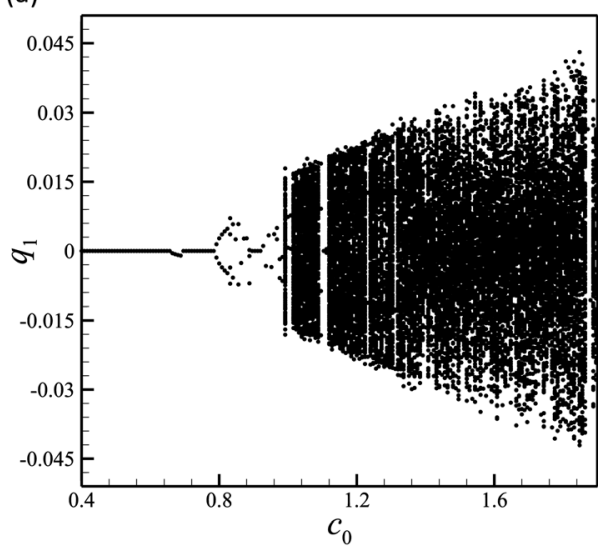

(c)

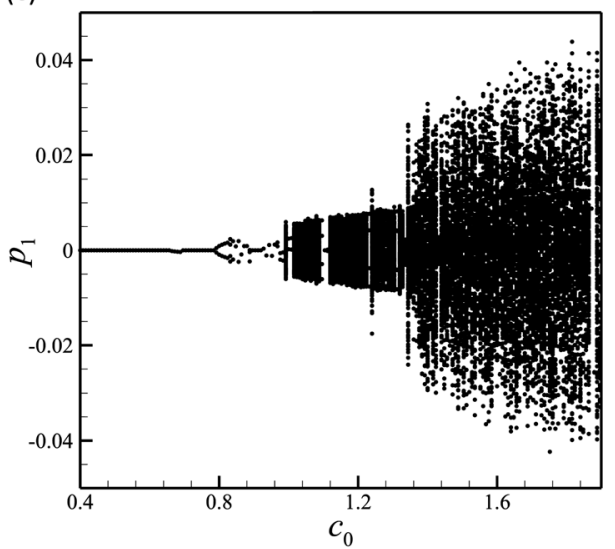

(e)

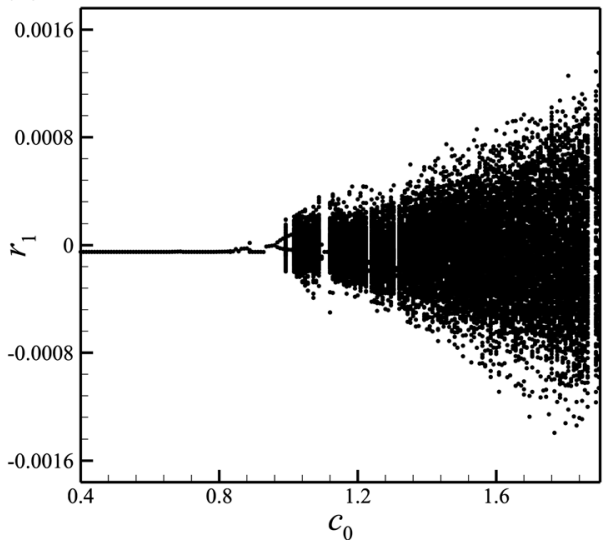

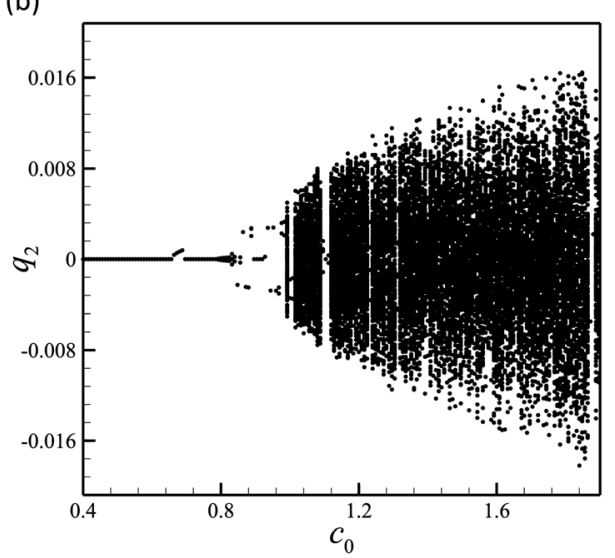

(d)

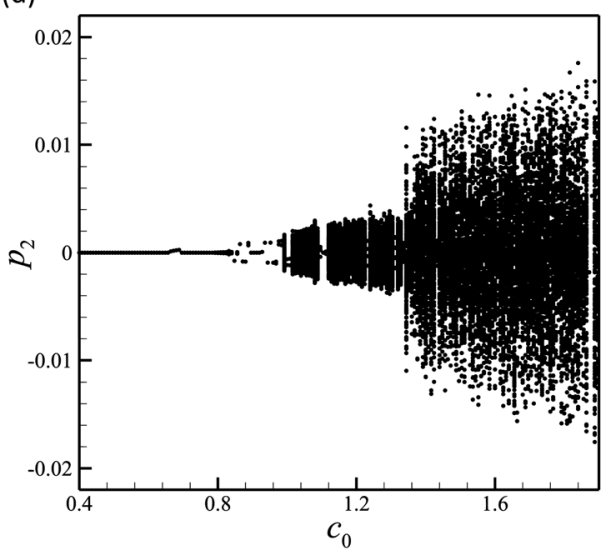

(f)

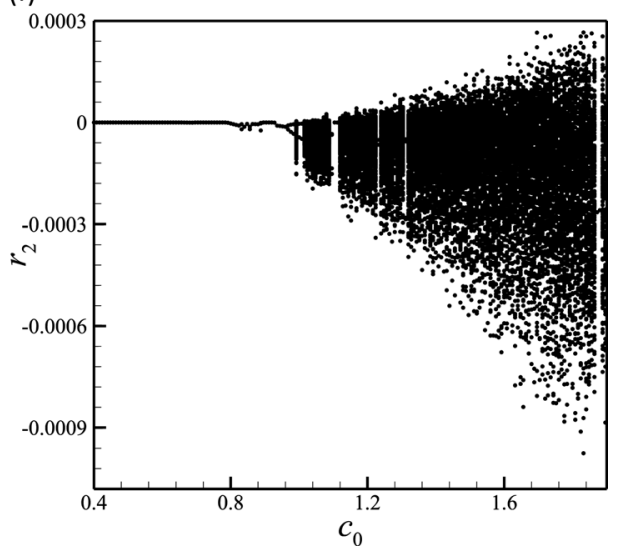

Fig. 13 Bifurcation diagrams of Poincaré points for increasing mean axial speed on the system with $c_{1}=0.40 ;((a)$ and $(b))$ the first two generalized coordinates of the transverse motion; $((c)$ and $(d))$ the first two generalized coordinates of the lateral motion; and $((e)$ and $(f))$ the first two generalized coordinates of the longitudinal motion 
(a)

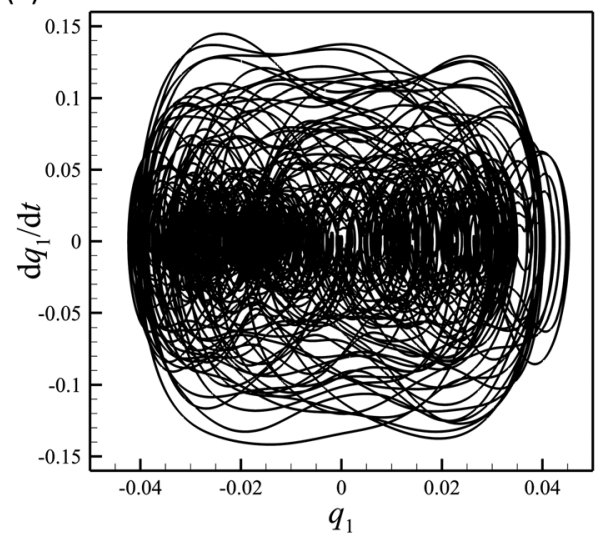

(c)

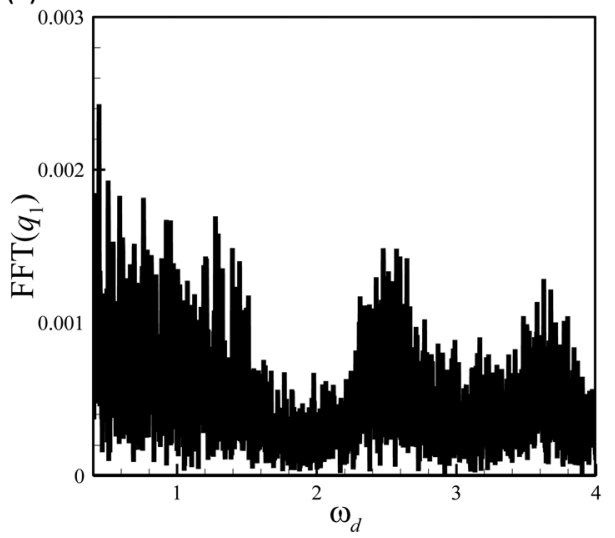

(b)

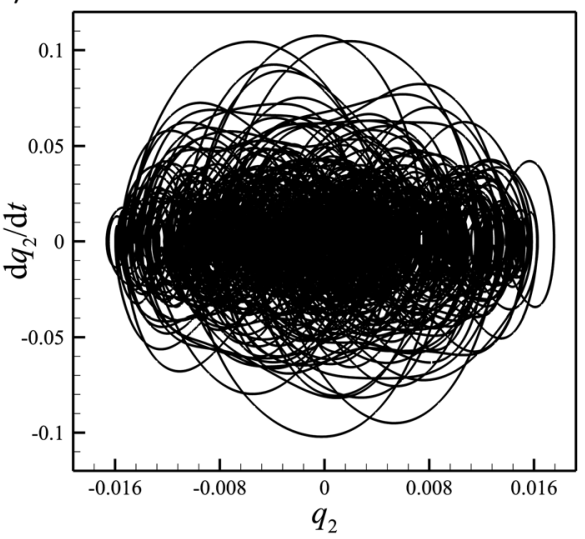

(d)

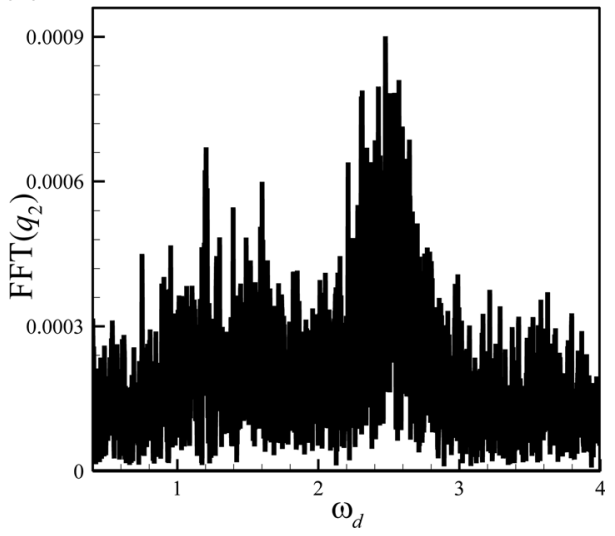

Fig. 14 Chaotic oscillations for the system of Fig. 13 at $c_{0}=1.848$ : $((a)$ and $(b))$ phase-plane diagrams of the $q_{1}$ and $q_{2}$ motions, respectively; and $((c)$ and $(d))$ FFTs of the $q_{1}$ and $q_{2}$ motions, respectively. $\omega_{\mathrm{d}}$ is the dimensionless frequency.

and 14, corresponding to the bifurcation diagrams of Figs. 11 and 13 , respectively.

\section{Conclusion}

The present study examined the 3D nonlinear dynamical behavior of an axially accelerating beam numerically. The nonlinear partial differential equations governing the motions of the system in the longitudinal, transverse, and lateral directions were obtained by means of Hamilton's principle. The discretized model of the system was obtained employing the Galerkin technique; in particular, the partial differential equations were recast into a set of first-order nonlinear ordinary differential equations with coupled nonlinear terms and time-dependent coefficients. A direct time integration was conducted upon this set of equations resulting in time-varying generalized coordinates. The bifurcation diagrams of Poincaré maps were then constructed by sectioning the phasespace in every period of the excitation frequency as either the amplitude of the axial speed variations or the mean value of the axial speed was increased as the bifurcation parameter.

The results for the case when the amplitude of the axial speed variations is selected as the bifurcation parameter revealed that: As a result of increased mean axial speed, in general, the chaotic regions increase substantially and the number of higher-order bifurcations decreases; at sufficiently large mean axial speeds, the system buckles to a new equilibrium configuration and oscillates over the buckled state.

Examining the global nonlinear dynamics of the system for the cases where the mean axial speed is varied as the bifurcation parameter showed that: In general, due to increased amplitude of the axial speed variation, the number of chaotic regions increases in the cascade of bifurcations and the chaotic regions become wider.
Moreover, the number of higher-order bifurcations decreases and the first bifurcation occurs at lower mean axial speeds.

\section{Acknowledgment}

The financial support to this research by the start-up grant of the University of Wollongong is gratefully acknowledged.

\section{References}

[1] Chen, L.-Q., and Wang, B., 2009, "Stability of Axially Accelerating Viscoelastic Beams: Asymptotic Perturbation Analysis and Differential Quadrature Validation," Eur. J. Mech.-A/Solids, 28(4), pp. 786-791.

[2] Ding, H., and Chen, L.-Q., 2010, "Galerkin Methods for Natural Frequencies of High-Speed Axially Moving Beams," J. Sound Vib., 329(17), pp. 3484-3494.

[3] Kong, L., and Parker, R. G., 2004, "Approximate Eigensolutions of Axially Moving Beams With Small Flexural Stiffness," J. Sound Vib., 276(1-2), pp. 459-469.

[4] Stylianou, M., and Tabarrok, B., 1994, "Finite Element Analysis of an Axially Moving Beam, Part I: Time Integration,” J. Sound Vib., 178(4), pp. 433-453.

[5] Pakdemirli, M., and Ulsoy, A. G., 1997, "Stability Analysis of an Axially Accelerating String," J. Sound Vib., 203(5), pp. 815-832.

[6] Pakdemirli, M., Ulsoy, A. G., and Ceranoglu, A., 1994, "Transverse Vibration of an Axially Accelerating String," J. Sound Vib., 169(2), pp. 179-196.

[7] Ding, H., Yan, Q.-Y., and Zu, J. W., 2014, "Chaotic Dynamics of an Axially Accelerating Viscoelastic Beam in the Supercritical Regime," Int. J. Bifurcation Chaos Appl. Sci. Eng., 24(5), p. 1450062.

[8] Yan, Q.-Y., Ding, H., and Chen, L.-Q., 2014, "Periodic Responses and Chaotic Behaviors of an Axially Accelerating Viscoelastic Timoshenko Beam," Nonlinear Dyn., 78(2), pp. 1577-1591.

[9] Ghayesh, M. H., Kafiabad, H. A., and Reid, T., 2012, "Sub- and Super-Critical Nonlinear Dynamics of a Harmonically Excited Axially Moving Beam,' Int. J. Solids Struct., 49(1), pp. 227-243.

[10] Ghayesh, M. H., 2011, "Nonlinear Forced Dynamics of an Axially Moving Viscoelastic Beam With an Internal Resonance," Int. J. Mech. Sci., 53(11), pp. 1022-1037.

[11] Ghayesh, M. H., Yourdkhani, M., Balar, S., and Reid, T., 2010, "Vibrations and Stability of Axially Traveling Laminated Beams," Appl. Math. Comput., 217(2), pp. 545-556. 
[12] Sze, K. Y., Chen, S. H., and Huang, J. L., 2005, "The Incremental Harmonic Balance Method for Nonlinear Vibration of Axially Moving Beams," J. Sound Vib., 281(3-5), pp. 611-626.

[13] Huang, J. L., Su, R. K. L., Li, W. H., and Chen, S. H., 2011, "Stability and Bifurcation of an Axially Moving Beam Tuned to Three-to-One Internal Resonances," J. Sound Vib., 330(3), pp. 471-485.

[14] Wickert, J. A., 1992, "Non-Linear Vibration of a Traveling Tensioned Beam," Int. J. Non-Linear Mech., 27(3), pp. 503-517.

[15] Marynowski, K., and Kapitaniak, T., 2007, "Zener Internal Damping in Modelling of Axially Moving Viscoelastic Beam With Time-Dependent Tension," Int. J. Non-Linear Mech., 42(1), pp. 118-131.

[16] Chen, L.-Q., and Yang, X.-D., 2006, "Vibration and Stability of an Axially Moving Viscoelastic Beam With Hybrid Supports," Eur. J. Mech.-A/Solids, 25(6), pp. 996-1008.

[17] Riedel, C. H., and Tan, C. A., 2002, "Coupled, Forced Response of an Axially Mov ing Strip With Internal Resonance," Int. J. Non-Linear Mech., 37(1), pp. 101-116.

[18] Ghayesh, M., 2012, "Stability and Bifurcations of an Axially Moving Beam With an Intermediate Spring Support," Nonlinear Dyn., 69(1-2), pp. 193-210.

[19] Suweken, G., and Van Horssen, W. T., 2003, "On the Transversal Vibrations of a Conveyor Belt With a Low and Time-Varying Velocity. Part II: The BeamLike Case," J. Sound Vib., 267(5), pp. 1007-1027.

[20] Öz, H. R., Pakdemirli, M., and Özkaya, E., 1998, "Transition Behaviour From String to Beam for an Axially Accelerating Material," J. Sound Vib., 215(3), pp. 571-576.

[21] Ozkaya, E., and Pakdemirli, M., 2000, "Vibrations of an Axially Accelerating Beam With Small Flexural Stiffness,” J. Sound Vib., 234(3), pp. 521-535.

[22] Pakdemirli, M., and Öz, H. R., 2008, "Infinite Mode Analysis and Truncation to Resonant Modes of Axially Accelerated Beam Vibrations," J. Sound Vib. 311(3-5), pp. 1052-1074.

[23] Xu, G. Y., and Zhu, W. D., 2010, "Nonlinear and Time-Varying Dynamics of High-Dimensional Models of a Translating Beam With a Stationary Load Subsystem," ASME J. Vib. Acoust., 132(6), p. 061012.

[24] Ghayesh, M. H., 2012, "Subharmonic Dynamics of an Axially Accelerating Beam,” Arch. Appl. Mech., 82(9), pp. 1169-1181.

[25] Ghayesh, M. H., 2012, "Coupled Longitudinal-Transverse Dynamics of an Axially Accelerating Beam," J. Sound Vib., 331(23), pp. 5107-5124.

[26] Öz, H. R., Pakdemirli, M., and Boyac1, H., 2001, "Non-Linear Vibrations and Stability of an Axially Moving Beam With Time-Dependent Velocity," Int J. Non-Linear Mech., 36(1), pp. 107-115.
[27] Pakdemirli, M., 2001, "A General Solution Procedure for Coupled Systems With Arbitrary Internal Resonances," Mech. Res. Commun., 28(6), pp. 617-622.

[28] Pakdemirli, M., and Boyac1, H., 2003, "Non-Linear Vibrations of a SimpleSimple Beam With a Non-Ideal Support in Between," J. Sound Vib., 268(2), pp. 331-341.

[29] Pakdemirli, M., and Özkaya, E., 2003, “Three-to-One Internal Resonances in a General Cubic Non-Linear Continuous System," J. Sound Vib., 268(3), pp. 543-553.

[30] Ghayesh, M., 2014, "Nonlinear Size-Dependent Behaviour of Single-Walled Carbon Nanotubes," Appl. Phys. A, 117(3), pp. 1393-1399.

[31] Ghayesh, M. H., 2012, "Nonlinear Dynamic Response of a Simply-Supported Kelvin-Voigt Viscoelastic Beam, Additionally Supported by a Nonlinear Spring," Nonlinear Anal.: Real World Appl., 13(3), pp. 1319-1333.

[32] Chen, L.-Q., Ding, H., and Lim, C. W., 2012, "Principal Parametric Resonance of Axially Accelerating Viscoelastic Beams: Multi-Scale Analysis and Differential Quadrature Verification," Shock Vib., 19(4), pp. 527-543.

[33] Chen, L.-Q., Tang, Y.-Q., and Lim, C. W., 2010, "Dynamic Stability in Parametric Resonance of Axially Accelerating Viscoelastic Timoshenko Beams," J. Sound Vib., 329(5), pp. 547-565.

[34] Chen, L.-Q., and Tang, Y.-Q., 2011, "Combination and Principal Parametric Resonances of Axially Accelerating Viscoelastic Beams: Recognition of Longitudinally Varying Tensions," J. Sound Vib., 330(23), pp. 5598-5614.

[35] Ghayesh, M. H., 2011, "On the Natural Frequencies, Complex Mode Functions, and Critical Speeds of Axially Traveling Laminated Beams: Parametric Study," Acta Mech. Solida Sin., 24(4), pp. 373-382.

[36] Ghayesh, M. H., Kazemirad, S., and Darabi, M. A., 2011, "A General Solution Procedure for Vibrations of Systems With Cubic Nonlinearities and Nonlinear/ Time-Dependent Internal Boundary Conditions,” J. Sound Vib., 330(22), pp. 5382-5400.

[37] Gholipour, A., Farokhi, H., and Ghayesh, M., 2014, "In-Plane and Out-of-Plane Nonlinear Size-Dependent Dynamics of Microplates," Nonlinear Dyn., 79(3), pp. 1771-1785.

[38] Ghayesh, M. H., and Farokhi, H., 2015, "Nonlinear Dynamics of Microplates," Int. J. Eng. Sci., 86, pp. 60-73.

[39] Farokhi, H., and Ghayesh, M. H., 2015, "Nonlinear Dynamical Behaviour of Geometrically Imperfect Microplates Based on Modified Couple Stress Theory,” Int. J. Mech. Sci., 90, pp. 133-144. 